\title{
Effects of diet form and type on growth performance, carcass yield, and iodine value of finishing pigs 1
}

\author{
J. E. Nemechek, * M. D. Tokach, ${ }^{*}$ S. S. Dritz, $\uparrow$ R. D. Goodband, $* 2$ \\ J. M. DeRouchey, and J. C. Woodworth* \\ *Department of Animal Sciences and Industry, College of Agriculture; and †Department of Diagnostic Medicine/ \\ Pathobiology, College of Veterinary Medicine, Kansas State University, Manhattan 66506-0201
}

\begin{abstract}
Two experiments were conducted to determine the effects of pelleting, diet type (fat and fiber level), and withdrawal of dietary fiber and fat before marketing on growth performance, carcass yield, and carcass fat iodine value (IV) of finishing pigs. Each experiment used 288 pigs (initially 49.6 and $48.5 \mathrm{~kg}$ $\mathrm{BW}$, respectively) with 6 dietary treatments arranged as $2 \times 3$ factorials. In Exp. 1, main effects were diet form (meal vs. pellet) and diet regimen. Diet regimens were 1) a low-fiber, low-fat (corn-soybean meal) diet from d 0 to 81,2$)$ a high-fiber, high-fat $(30 \%$ dried distillers grains with solubles [DDGS] and 19\% wheat middlings [midds]) diet from d 0 to 64 followed by the low-fiber, low-fat diet from d 64 to 81 (fiber and fat withdrawal), and 3) the high-fiber, high-fat diet fed from d 0 to 81 . Pigs fed pelleted diets had increased $(P<0.05)$ ADG and G:F compared with those fed meal diets. Pigs fed pelleted diets had increased belly fat IV $(2.9 \mathrm{mg} / \mathrm{g})$ compared with those fed meal diets, with a greater increase when fed high-fiber, high-fat diets throughout the entire study (interaction, $P<0.05$ ). Pigs fed the low-fiber, low-fat diet throughout had increased $(P<$ $0.001) \mathrm{G}: F$ compared with pigs fed the other 2 treat-
\end{abstract}

ments. Pigs fed low-fiber, low-fat diets throughout the study or pigs withdrawn from high-fiber, high-fat diets had increased $(P<0.001)$ carcass yield compared with pigs fed high-fiber, high-fat diets throughout. In Exp. 2, treatment main effects were diet form (meal vs. pellet) and diet type (corn-soybean meal-based control, the control with 30\% DDGS and 19\% midds, or the control diet with 3\% corn oil). The diet containing corn oil was calculated to produce carcass fat IV similar to diets containing DDGS and midds. Overall, pigs fed pelleted diets had increased $(P<0.05)$ ADG, G:F, and belly fat IV $(1.3 \mathrm{mg} / \mathrm{g})$ compared with those fed meal diets. Pigs fed the diets containing DDGS and midds had decreased $(P<0.05)$ ADG, carcass yield, and HCW compared with pigs fed the control or corn oil diets and decreased $(P<0.001) \mathrm{G}$ :F compared with pigs fed added corn oil. Belly IV was greatest $(P<0.001)$ for pigs fed diets with DDGS and midds and lowest for pigs fed the control diet, with pigs fed the corn oil diets intermediate. In conclusion, pelleting diets improves pig ADG (approximately 3\%) and G:F (approximately 6\%); however, a novel finding of this study is that pelleting diets fed to finishing pigs also increases belly fat IV.

Key words: carcass yield, corn oil, distillers dried grains with solubles, finishing pig, pelleting

(C) 2015 American Society of Animal Science. All rights reserved.

J. Anim. Sci. 2015.93:4486-4499

doi:10.2527/jas2015-9149

\section{INTRODUCTION}

Studies have observed that up to $30 \%$ dried distillers grains with solubles (DDGS) can be fed without negative effects on growth performance (Widmer et al., 2007; Stein and Shurson, 2009; Xu et al., 2010).

${ }^{1}$ Contribution number 15-277-J from the Kansas Agricultural When DDGS were combined with wheat middlings (midds), however, Salyer et al. (2012) observed linear decreases in $\mathrm{ADG}$ and G:F as midds were added at $0 \%, 10 \%$, or $20 \%$. 
In addition, feeding high levels of DDGS, mid$\mathrm{ds}$, or the combination of both reduces carcass yield of finishing pigs (Linneen et al., 2008; Salyer et al., 2012). This is due to increased large intestinal weights caused by the high-fiber component of the ingredients (Asmus et al., 2014). Along with the dietary fiber component from DDGS and midds, the oil content, particularly C18:2 fatty acids, also increases (NRC, 2012). Iodine value (IV), a measure of unsaturated fatty acids (UFA), is 1 method used by packers to assess fat quality in pork. Feeding ingredients high in UFA increases carcass fat IV (Benz et al., 2011), thus producing less desirable product for the meat packing industry (McClelland et al., 2012). Reducing or withdrawing added DDGS and midds before marketing can mitigate some of the negative effects on carcass yield and carcass fat IV (Gaines et al., 2007; Asmus et al., 2014; Coble et al., 2014).

The beneficial effects of pelleting swine diets on growth performance of finishing pigs also have been documented, including increased BW gain and improved feed efficiency (Baird, 1973; Wondra et al., 1995), but most of the previous pelleting research has evaluated corn-soybean meal-based diets with limited inclusion of by-products. In addition, to our knowledge, the effects of pelleting on FA profile and IV of carcass fat have not been reported. Therefore, the objective of these trials was to determine the effects of diet type and form on growth performance, carcass yield, and carcass fat IV of finishing pigs.

\section{MATERIALS AND METHODS}

All experimental procedures and animal care were approved by the Kansas State University Institutional Animal Care and Use Committee.

\section{General}

Experiments 1 and 2 each used a total of 288 finishing pigs $(327 \times 1050$, PIC, Hendersonville, TN $)$ initially 49.6 and $48.5 \mathrm{~kg}$ BW, respectively. Pigs were housed in a totally enclosed, environmentally regulated, mechanically ventilated barn containing 36 pens $(2.44 \times 3.05 \mathrm{~m})$. The pens had adjustable gates facing the alleyway and allowed $0.93 \mathrm{~m}^{2} / \mathrm{pig}$. Each pen was equipped with a cup waterer and a single-sided, dry self-feeder (Farmweld, Teutopolis, IL) with 2 eating spaces located in the fence line. Pens were located over a completely slatted concrete floor with a $1.20-\mathrm{m}$ pit underneath for manure storage. All pigs were provided ad libitum access to feed and water.

Each experiment was arranged as a $2 \times 3$ factorial. Pens were randomly allotted to 1 of 6 experimen- tal treatments with 6 pens per treatment and 8 pigs per pen ( 4 barrows and 4 gilts per pen). Pigs and feeders were weighed approximately every $2 \mathrm{wk}$ to calculate ADG, ADFI, G:F, and caloric efficiency on a ME and NE basis. Diets for both experiments were prepared and pelleted at a commercial feed mill in Beloit, KS (Hubbard Feeds Inc., Mankato, MN). All pelleted diets were processed with a Sprout Waldron Pellet Mill (model Ace 501; Sprout, Waldron \& Company, Inc., Muncy, PA) equipped with a 4.37-mm-diam. die. Before pelleting, diets were conditioned with steam at $71^{\circ} \mathrm{C}$ for approximately $20 \mathrm{~s}$. A single batch of meal feed was prepared; half was fed as such, and half was pelleted. Diets were delivered to feeders using a computerized feeding system (FeedPro, Feedlogic Corp., Willmar, MN) that recorded all feed additions. Feed samples were taken at the feeder during each phase. All diets were analyzed for moisture (method 934.01; AOAC, 2006), CP (method 990.03; AOAC, 2006), ether extract (method 920.39 A; AOAC, 2006), crude fiber (method 978.10; AOAC, 2006), ADF, and NDF. Pellet durability index (PDI) was determined using the standard tumbling-box technique (standard S269.4; American Society for Agricultural Engineers [ASAE], 1996), and modified PDI was done by adding 5 hexagonal nuts $(1.27 \mathrm{~cm})$ before tumbling. Percentage of fines (ASAE, 1987) was also measured in duplicate for all pelleted diets, with fines characterized as material that would pass through a \#6 sieve (3,360$\mu \mathrm{m}$ openings). All pellet quality measurements were analyzed at the K-State O. H. Kruse Feed Technology Innovation Center.

At the end of each trial, pigs were individually tattooed in sequential order to allow for carcass data collection at a commercial packing plant and data retrieval by pig. Hot carcass weights were measured immediately after evisceration and were used to calculate percentage yield by dividing HCW at the plant by live weight at the farm before transport. All carcass fat samples were collected from the pig's left side. For both experiments, belly fat samples were collected from the ventral side of the belly along the navel edge between the 10th and the 12th ribs of each pig. In Exp. 2, fat samples were also collected from the shoulder of each pig approximately $5 \mathrm{~cm}$ dorsal to the medial ridge of the scapula. All fat samples were immediately frozen after collection and remained frozen until preparation for FA analysis could be conducted. Fat samples were thawed, and adipose tissue was isolated by removing the skin and lean tissue. Samples were then analyzed for FA profiles using gas chromatography as described in detail by Asmus et al. (2014). Iodine value was calculated using the following equation (American Oil Chemists' Society, 1998): IV = $[\mathrm{C} 16: 1] \times 0.95+[\mathrm{C} 18: 1] \times 0.86+[\mathrm{C} 18: 2] \times 1.732+$ $[\mathrm{C} 18: 3] \times 2.616+[\mathrm{C} 20: 1] \times 0.785+[\mathrm{C} 22: 1] \times 0.723$. 
Table 1. Composition of diets, Exp. 1 (as-fed basis) ${ }^{1}$

\begin{tabular}{|c|c|c|c|c|c|c|c|c|}
\hline \multirow[b]{2}{*}{ Item } & \multicolumn{2}{|c|}{ Phase 1} & \multicolumn{2}{|c|}{ Phase 2} & \multicolumn{2}{|c|}{ Phase 3} & \multicolumn{2}{|c|}{ Phase 4} \\
\hline & Low fiber & High fiber & Low fiber & High fiber & Low fiber & High fiber & Low fiber & Highfiber \\
\hline \multicolumn{9}{|l|}{ Ingredient, \% } \\
\hline Corn & 73.71 & 34.88 & 78.93 & 39.99 & 82.65 & 43.56 & 84.97 & 45.79 \\
\hline Soybean meal, $46.5 \%$ CP & 23.80 & 13.74 & 18.84 & 8.71 & 15.32 & 5.20 & 13.15 & 3.04 \\
\hline Dried distillers grains with solubles & - & 30.00 & - & 30.00 & - & 30.00 & - & 30.00 \\
\hline Wheat middlings & - & 19.00 & - & 19.00 & - & 19.00 & - & 19.00 \\
\hline Monocalcium P, 21\% P & 0.45 & - & 0.35 & - & 0.25 & - & 0.20 & - \\
\hline Limestone & 1.05 & 1.30 & 1.00 & 1.28 & 0.98 & 1.29 & 0.93 & 1.28 \\
\hline Salt & 0.35 & 0.35 & 0.35 & 0.35 & 0.35 & 0.35 & 0.35 & 0.35 \\
\hline Vitamin premix ${ }^{2}$ & 0.15 & 0.15 & 0.13 & 0.13 & 0.10 & 0.10 & 0.08 & 0.08 \\
\hline Trace mineral premix ${ }^{3}$ & 0.15 & 0.15 & 0.13 & 0.13 & 0.10 & 0.10 & 0.08 & 0.08 \\
\hline L-Lys $\cdot \mathrm{HCl}$ & 0.170 & 0.310 & 0.150 & 0.293 & 0.135 & 0.278 & 0.128 & 0.270 \\
\hline DL-Met & 0.020 & - & - & - & - & - & - & - \\
\hline L-Thr & 0.025 & - & 0.010 & - & - & - & - & - \\
\hline Phytase $^{4}$ & 0.125 & 0.125 & 0.125 & 0.125 & 0.125 & 0.125 & 0.125 & 0.125 \\
\hline Total & 100.0 & 100.0 & 100.0 & 100.0 & 100.0 & 100.0 & 100.0 & 100.0 \\
\hline \multicolumn{9}{|l|}{ Calculated analysis } \\
\hline \multicolumn{9}{|c|}{ Standardized ileal digestible amino acids, $\%$} \\
\hline Lys & 0.93 & 0.93 & 0.79 & 0.79 & 0.69 & 0.69 & 0.63 & 0.63 \\
\hline Ile:Lys & 69 & 72 & 70 & 74 & 72 & 76 & 73 & 78 \\
\hline Met:Lys & 30 & 34 & 30 & 37 & 32 & 40 & 33 & 43 \\
\hline Met + Cys:Lys & 59 & 70 & 62 & 77 & 66 & 83 & 69 & 88 \\
\hline Thr:Lys & 63 & 66 & 63 & 69 & 64 & 72 & 66 & 74 \\
\hline Trp:Lys & 19 & 19 & 19 & 19 & 19 & 19 & 19 & 19 \\
\hline Val:Lys & 78 & 88 & 81 & 94 & 85 & 99 & 87 & 103 \\
\hline Total Lys, \% & 1.04 & 1.09 & 0.89 & 0.94 & 0.78 & 0.83 & 0.72 & 0.77 \\
\hline $\mathrm{ME}, \mathrm{kcal} / \mathrm{kg}$ & 3,296 & 3,233 & 3,307 & 3,240 & 3,316 & 3,245 & 3,324 & 3,249 \\
\hline $\mathrm{NE}, \mathrm{kcal} / \mathrm{kg}$ & 2,474 & 2,333 & 2,507 & 2,365 & 2,533 & 2,386 & 2,549 & 2,400 \\
\hline $\mathrm{CP}, \%$ & 17.5 & 20.8 & 15.6 & 18.9 & 14.3 & 17.6 & 13.5 & 16.7 \\
\hline $\mathrm{Ca}, \%$ & 0.59 & 0.58 & 0.53 & 0.56 & 0.49 & 0.55 & 0.46 & 0.54 \\
\hline $\mathrm{P}, \%$ & 0.47 & 0.58 & 0.42 & 0.56 & 0.39 & 0.55 & 0.37 & 0.54 \\
\hline Available P, \% & 0.27 & 0.39 & 0.25 & 0.38 & 0.22 & 0.38 & 0.21 & 0.37 \\
\hline
\end{tabular}

${ }^{1}$ Phase 1 diets were fed from d 0 to 15 , phase 2 from d 15 to 40 , phase 3 from d 40 to 64 , and phase 4 from d 64 to 81 . Each diet was fed in either meal or pellet form.

${ }^{2}$ Provided per kilogram of premix: 4,409,200 IU vitamin A, 551,150 IU vitamin $\mathrm{D}_{3}, 17,637 \mathrm{IU}$ vitamin E, 1,764 mg vitamin K, 3,307 mg riboflavin, $11,023 \mathrm{mg}$ pantothenic acid, $19,841 \mathrm{mg}$ niacin, and $15.4 \mathrm{mg}$ vitamin $\mathrm{B}_{12}$.

${ }^{3}$ Provided per kilogram of premix: $26.5 \mathrm{~g}$ Mn from manganese oxide, $110 \mathrm{~g}$ Fe from iron sulfate, $110 \mathrm{~g} \mathrm{Zn}$ from zinc sulfate, $11 \mathrm{~g}$ Cu from copper sulfate, $198 \mathrm{mg}$ I from calcium iodate, and $198 \mathrm{mg}$ Se from sodium selenite.

${ }^{4}$ Phyzyme 600 (Danisco Animal Nutrition, St. Louis, MO) provided 780 phytase units (FTU)/kg, with a release of $0.11 \%$ available P.

\section{Experiment 1}

An 81-d trial was conducted to determine the effects of diet form and fiber withdrawal on growth performance, carcass yield, and carcass fat IV of finishing pigs. Treatment main effects included diet form (meal or pellet) and diet regimen. The 3 diet regimens were 1) low fiber, low fat (corn-soybean meal) from d 0 to 81,2) high fiber, high fat (30\% DDGS and 19\% midds) from d 0 to 64 followed by low fiber, low fat from d 64 to 81 (fiber and fat withdrawal), and 3) high fiber, high fat from d 0 to 81 (Table 1). Diets were fed in 4 phases from d 0 to 14,14 to 40,40 to 64 , and 64 to 81 . Diets within phase were formulated to contain equal amounts of stan- dardized ileal digestible (SID) Lys with 0.93, 0.79, 0.69, and 0.63 SID Lys for phases 1, 2, 3, and 4, respectively. Thus, diets contained different SID Lys:energy ratios as dietary energy was not held constant.

\section{Experiment 2}

An 87-d trial was conducted to determine the effects of diet form and type on growth performance, carcass yield, and carcass fat IV of finishing pigs. Treatments were arranged in a $2 \times 3$ factorial with the main effects of diet form and type. The 2 diet forms used were meal and pellet. The 3 dietary types were 1) corn-soybean meal-based control, 2) control with 
Table 2. Composition of phase 1 and 2 diets, Exp. 2 (as-fed basis)

\begin{tabular}{|c|c|c|c|c|c|c|}
\hline \multirow[b]{2}{*}{ Item } & \multicolumn{3}{|c|}{ Phase $1^{1}$} & \multicolumn{3}{|c|}{ Phase $2^{2}$} \\
\hline & Control & $\begin{array}{l}\text { DDGS + } \\
\text { midds }\end{array}$ & $\begin{array}{c}\text { Corn } \\
\text { oil }\end{array}$ & Control & $\begin{array}{l}\text { DDGS + } \\
\text { midds }\end{array}$ & $\begin{array}{c}- \text { Corn } \\
\text { oil }\end{array}$ \\
\hline \multicolumn{7}{|l|}{ Ingredient, \% } \\
\hline Corn & 72.01 & 33.03 & 68.84 & 77.57 & 37.46 & 74.25 \\
\hline $\begin{array}{c}\text { Soybean meal, } \\
46.5 \% \mathrm{CP}\end{array}$ & 25.56 & 15.70 & 25.64 & 20.17 & 11.36 & 20.40 \\
\hline DDGS & - & 30.00 & - & - & 30.00 & - \\
\hline Wheat middlings & - & 19.00 & - & - & 19.00 & - \\
\hline Corn oil & - & - & 3.00 & - & - & 3.00 \\
\hline $\begin{array}{l}\text { Monocalcium } \mathrm{P} \text {, } \\
21 \% \mathrm{P}\end{array}$ & 0.45 & - & 0.52 & 0.37 & - & 0.44 \\
\hline Limestone & 1.05 & 1.30 & 1.05 & 1.00 & 1.28 & 1.00 \\
\hline Salt & 0.35 & 0.35 & 0.35 & 0.35 & 0.35 & 0.35 \\
\hline Vitamin premix ${ }^{3}$ & 0.150 & 0.150 & 0.150 & 0.125 & 0.125 & 0.125 \\
\hline $\begin{array}{l}\text { Trace mineral } \\
\text { premix }^{4}\end{array}$ & 0.150 & 0.150 & 0.150 & 0.125 & 0.125 & 0.125 \\
\hline L-Lys $\cdot \mathrm{HCl}$ & 0.220 & 0.310 & 0.225 & 0.235 & 0.293 & 0.235 \\
\hline $\begin{array}{l}\text { Met hydroxyl } \\
\text { analog }\end{array}$ & 0.020 & - & 0.028 & 0.013 & - & 0.015 \\
\hline L-Thr & 0.030 & - & 0.040 & 0.035 & - & 0.040 \\
\hline Phytase $^{5}$ & 0.012 & 0.012 & 0.012 & 0.015 & 0.015 & 0.015 \\
\hline Total & 100.0 & 100.0 & 100.0 & 100.0 & 100.0 & 100.0 \\
\hline \multicolumn{7}{|l|}{ Calculated analysis } \\
\hline \multicolumn{7}{|c|}{ Standardized ileal digestible amino acids, $\%$} \\
\hline Lys & 0.98 & 0.98 & 0.98 & 0.86 & 0.86 & 0.86 \\
\hline Ile:Lys & 67 & 71 & 66 & 65 & 72 & 65 \\
\hline Met:Lys & 28 & 33 & 29 & 29 & 35 & 29 \\
\hline Met + Cys:Lys & 55 & 62 & 55 & 56 & 67 & 56 \\
\hline Thr:Lys & 60 & 62 & 61 & 61 & 63 & 61 \\
\hline Trp:Lys & 19 & 19 & 19 & 18 & 18 & 18 \\
\hline Val:Lys & 74 & 85 & 73 & 74 & 88 & 73 \\
\hline Total Lys, \% & 1.11 & 1.19 & 1.11 & 0.98 & 1.05 & 0.97 \\
\hline $\mathrm{ME}, \mathrm{kcal} / \mathrm{kg}$ & 3,298 & 3,234 & 3,452 & 3,309 & 3,241 & 3,463 \\
\hline $\mathrm{NE}, \mathrm{kcal} / \mathrm{kg}$ & 2,466 & 2,325 & 2,610 & 2,502 & 2,352 & 2,646 \\
\hline $\mathrm{CP}, \%$ & 18.4 & 21.7 & 18.1 & 16.3 & 20.0 & 16.1 \\
\hline $\mathrm{Ca}, \%$ & 0.55 & 0.57 & 0.56 & 0.50 & 0.55 & 0.52 \\
\hline $\mathrm{P}, \%$ & 0.47 & 0.56 & 0.47 & 0.42 & 0.54 & 0.43 \\
\hline Available P, \% & 0.29 & 0.37 & 0.30 & 0.26 & 0.37 & 0.28 \\
\hline
\end{tabular}

${ }^{1}$ Phase 1 diets were fed from d 0 to 21. DDGS $=$ dried distillers grains with solubles; midds $=$ wheat middlings.

${ }^{2}$ Phase 2 diets were fed from d 21 to 45 .

${ }^{3}$ Provided per kilogram of premix: 4,409,200 IU vitamin A, 551,150 IU vitamin $\mathrm{D}_{3}, 17,637 \mathrm{IU}$ vitamin $\mathrm{E}, 1,764 \mathrm{mg}$ vitamin $\mathrm{K}, 3,307 \mathrm{mg}$ riboflavin, $11,023 \mathrm{mg}$ pantothenic acid, $19,841 \mathrm{mg}$ niacin, and $15.4 \mathrm{mg}$ vitamin $\mathrm{B}_{12}$.

${ }^{4}$ Provided per kilogram of premix: $26.5 \mathrm{~g} \mathrm{Mn}$ from manganese oxide, $110 \mathrm{~g}$ Fe from iron sulfate, $110 \mathrm{~g} \mathrm{Zn}$ from zinc sulfate, $11 \mathrm{~g} \mathrm{Cu}$ from copper sulfate, $198 \mathrm{mg}$ I from calcium iodate, and $198 \mathrm{mg}$ Se from sodium selenite.

${ }^{5}$ Natuphos 2500 (BASF Corp., Mt. Olive, NJ) provided 300 phytase units (FTU) $/ \mathrm{kg}$, with a release of $0.10 \%$ available P.

$30 \%$ DDGS and 19\% midds, and 3) control with $3 \%$ corn oil (Tables 2 and 3). The corn-soybean mealbased control provided a baseline, whereas the diet containing 30\% DDGS and 19\% midds was the previously established diet from Exp. 1 that allowed for
Table 3. Composition of phase 3 and 4 diets, Exp. 2 (as-fed basis)

\begin{tabular}{|c|c|c|c|c|c|c|}
\hline \multirow[b]{2}{*}{ Item } & \multicolumn{3}{|c|}{ Phase $3^{1}$} & \multicolumn{3}{|c|}{ Phase $4^{2}$} \\
\hline & Control & $\begin{array}{l}\mathrm{DDGS}+ \\
\text { midds }\end{array}$ & $\begin{array}{c}\text { Corn } \\
\text { oil }\end{array}$ & Control & $\begin{array}{l}\text { DDGS + } \\
\text { midds }\end{array}$ & $\begin{array}{c}\text { corn } \\
\text { oil }\end{array}$ \\
\hline \multicolumn{7}{|l|}{ Ingredient, \% } \\
\hline Corn & 81.04 & 40.70 & 77.72 & 83.98 & 43.80 & 80.62 \\
\hline $\begin{array}{c}\text { Soybean meal } \\
(46.5 \% \mathrm{CP})\end{array}$ & 16.81 & 8.16 & 17.04 & 13.86 & 5.13 & 14.17 \\
\hline DDGS & 一 & 30.00 & - & - & 30.00 & - \\
\hline Wheat middlings & - & 19.00 & - & - & 19.00 & - \\
\hline Corn oil & - & - & 3.00 & - & - & 3.00 \\
\hline $\begin{array}{l}\text { Monocalcium } \mathrm{P} \\
21 \% \mathrm{P}\end{array}$ & 0.34 & - & 0.42 & 0.45 & - & 0.49 \\
\hline Limestone & 0.98 & 1.29 & 0.98 & 0.93 & 1.28 & 0.93 \\
\hline Salt & 0.35 & 0.35 & 0.35 & 0.35 & 0.35 & 0.35 \\
\hline Vitamin premix ${ }^{3}$ & 0.100 & 0.100 & 0.100 & 0.075 & 0.075 & 0.075 \\
\hline $\begin{array}{l}\text { Trace mineral } \\
\text { premix }^{4}\end{array}$ & 0.100 & 0.100 & 0.100 & 0.075 & 0.075 & 0.075 \\
\hline L-Lys· $\cdot \mathrm{HCl}$ & 0.225 & 0.278 & 0.225 & 0.215 & 0.270 & 0.213 \\
\hline $\begin{array}{l}\text { Met hydroxyl } \\
\text { analog }\end{array}$ & 0.010 & - & 0.010 & - & - & 0.010 \\
\hline L-Thr & 0.038 & - & 0.043 & 0.050 & - & 0.055 \\
\hline Phytase $^{5}$ & 0.018 & 0.018 & 0.018 & 0.021 & 0.021 & 0.021 \\
\hline Total & 100.0 & 100.0 & 100.0 & 100.0 & 100.0 & 100.0 \\
\hline \multicolumn{7}{|l|}{ Calculated analysis } \\
\hline \multicolumn{7}{|c|}{ Standardized ileal digestible amino acids, $\%$} \\
\hline Lys & 0.77 & 0.77 & 0.77 & 0.69 & 0.69 & 0.69 \\
\hline Ile:Lys & 66 & 74 & 65 & 66 & 75 & 66 \\
\hline Met:Lys & 30 & 37 & 29 & 30 & 40 & 31 \\
\hline Met + Cys:Lys & 59 & 71 & 58 & 60 & 75 & 61 \\
\hline Thr:Lys & 62 & 65 & 62 & 65 & 67 & 66 \\
\hline Trp:Lys & 18 & 18 & 18 & 18 & 18 & 18 \\
\hline Val:Lys & 75 & 92 & 74 & 77 & 96 & 76 \\
\hline Total Lys, $\%$ & 0.88 & 0.96 & 0.88 & 0.79 & 0.87 & 0.79 \\
\hline $\mathrm{ME}, \mathrm{kcal} / \mathrm{kg}$ & 3,316 & 3,245 & 3,470 & 3,318 & 3,250 & 3,472 \\
\hline $\mathrm{NE}, \mathrm{kcal} / \mathrm{kg}$ & 2,524 & 2,372 & 2,668 & 2,542 & 2,392 & 2,685 \\
\hline $\mathrm{CP}, \%$ & 14.9 & 18.7 & 14.8 & 13.8 & 17.5 & 13.6 \\
\hline $\mathrm{Ca}, \%$ & 0.48 & 0.54 & 0.49 & 0.47 & 0.53 & 0.48 \\
\hline $\mathrm{P}, \%$ & 0.40 & 0.53 & 0.41 & 0.41 & 0.52 & 0.41 \\
\hline Available P, \% & 0.25 & 0.36 & 0.27 & 0.27 & 0.36 & 0.28 \\
\hline
\end{tabular}

${ }^{1}$ Phase 3 diets were fed from d 45 to 70. DDGS $=$ dried distillers grains with solubles; midds $=$ wheat middlings.

${ }^{2}$ Phase 4 diets were fed from d 70 to 87 .

${ }^{3}$ Provided per kilogram of premix: 4,409,200 IU vitamin A, 551,150 IU vitamin $\mathrm{D}_{3}, 17,637 \mathrm{IU}$ vitamin $\mathrm{E}, 1,764 \mathrm{mg}$ vitamin $\mathrm{K}, 3,307 \mathrm{mg}$ riboflavin, $11,023 \mathrm{mg}$ pantothenic acid, $19,841 \mathrm{mg}$ niacin, and $15.4 \mathrm{mg}$ vitamin $\mathrm{B}_{12}$.

${ }^{4}$ Provided per kilogram of premix: $26.5 \mathrm{~g} \mathrm{Mn}$ from manganese oxide, $110 \mathrm{~g}$ Fe from iron sulfate, $110 \mathrm{~g} \mathrm{Zn}$ from zinc sulfate, $11 \mathrm{~g} \mathrm{Cu}$ from copper sulfate, $198 \mathrm{mg}$ I from calcium iodate, and $198 \mathrm{mg}$ Se from sodium selenite.

${ }^{5}$ Natuphos 2500 (BASF Corp., Mt. Olive, NJ) provided 300 phytase units $(\mathrm{FTU}) / \mathrm{kg}$, with a release of $0.10 \%$ available P.

a predic increase in carcass fat IV. Extracted corn oil was used in the third treatment to compare it to the endogenous corn oil present in the DDGS. The level of $3 \%$ corn oil was selected on the basis of research conducted by Benz et al. (2011) with soybean oil in an 
Table 4. Chemical analysis of diets, Exp. $1^{1}$

\begin{tabular}{|c|c|c|c|c|c|c|c|c|}
\hline \multirow[b]{3}{*}{ Item } & \multicolumn{4}{|c|}{ Phase $1^{2}$} & \multicolumn{4}{|c|}{ Phase $2^{3}$} \\
\hline & \multicolumn{2}{|c|}{ Meal } & \multicolumn{2}{|c|}{ Pellet } & \multicolumn{2}{|c|}{ Meal } & \multicolumn{2}{|c|}{ Pellet } \\
\hline & $\begin{array}{l}\text { Low } \\
\text { fiber }\end{array}$ & $\begin{array}{l}\text { High } \\
\text { fiber }\end{array}$ & $\begin{array}{l}\text { Low } \\
\text { fiber }\end{array}$ & $\begin{array}{l}\text { High } \\
\text { fiber }\end{array}$ & $\begin{array}{l}\text { Low } \\
\text { fiber }\end{array}$ & $\begin{array}{l}\text { High } \\
\text { fiber }\end{array}$ & $\begin{array}{l}\text { Low } \\
\text { fiber }\end{array}$ & $\begin{array}{l}\text { High } \\
\text { fiber }\end{array}$ \\
\hline DM, \% & 89.58 & 89.96 & 89.71 & 89.06 & 90.58 & 88.60 & 90.07 & 88.54 \\
\hline $\mathrm{CP}, \%$ & 18.1 & 20.6 & 18.4 & 22.1 & 16.8 & 21.3 & 17.0 & 20.8 \\
\hline $\mathrm{ADF}, \%$ & 3.1 & 7.1 & 3.1 & 5.9 & 2.5 & 7.0 & 1.9 & 4.9 \\
\hline NDF, \% & 5.7 & 14.5 & 6 & 14.2 & 6.0 & 15.5 & 5.9 & 13.9 \\
\hline $\begin{array}{l}\text { Crude } \\
\quad \text { fiber, \% }\end{array}$ & 1.8 & 4.0 & 1.9 & 3.5 & 1.7 & 4.3 & 1.8 & 3.5 \\
\hline $\begin{array}{l}\text { Ether } \\
\text { extract, } \\
\%\end{array}$ & 1.6 & 3.7 & 1.7 & 4.0 & 2.3 & 4.7 & 2.0 & 4.5 \\
\hline $\mathrm{Ca}, \%$ & 0.55 & 0.63 & 0.56 & 0.66 & 0.39 & 0.60 & 0.42 & 0.61 \\
\hline $\mathrm{P}, \%$ & 0.46 & 0.65 & 0.47 & 0.63 & 0.45 & 0.69 & 0.43 & 0.59 \\
\hline
\end{tabular}

${ }^{1} \mathrm{~A}$ composite sample consisting of 6 subsamples was used for analysis.

${ }^{2}$ Phase 1 diets were fed from $\mathrm{d} 0$ to 15 .

${ }^{3}$ Phase 2 diets were fed from $\mathrm{d} 15$ to 40 .

effort to obtain carcass fat IV similar to that of pigs fed the diet containing DDGS and midds. Diets were fed in 4 phases from d 0 to 21,21 to 45,45 to 70 , and 70 to 87. Diets within phase were formulated to contain equal amounts of SID Lys with $0.98,0.86,0.77$, and 0.69 SID Lys for phases 1, 2, 3, and 4, respectively.

\section{Statistical Analysis}

Experimental data for both trials were analyzed using ANOVA as a $2 \times 3$ factorial using the MIXED procedure of SAS (SAS Inst. Inc., Cary, NC). Pen was the experimental unit for all data analysis. For HCW, carcass yield, and carcass fat IV, measurements were collected for each pig; then pen means were calculated and used in the model. Experiment 1 included main effects of 2 diet forms and 3 diet regimens and their interaction as fixed effects, and Exp. 2 included main effects of 2 diet forms and 3 diet types and their interactions. Differences between treatments were determined using least squares means with results considered significant at $P \leq 0.05$ and a trend at $P \leq 0.10$.

\section{RESULTS}

\section{Experiment 1}

Chemical Analysis and Pellet Quality Measurements. Analysis of diets revealed that as expected, the inclusion of dietary DDGS and midds increased ADF, NDF, crude fiber, and ether extract (Tables 4 and 5). Standard PDI was greater than $90 \%$ during all phases for pelleted diets (Table 6). Percentage of fines was low for all diets and phases at less than $10 \%$ fines.
Table 5. Chemical analysis of diets, Exp. $1^{1}$

\begin{tabular}{|c|c|c|c|c|c|c|c|c|}
\hline \multirow[b]{3}{*}{ Item } & \multicolumn{4}{|c|}{ Phase $3^{2}$} & \multicolumn{4}{|c|}{ Phase $4^{3}$} \\
\hline & \multicolumn{2}{|c|}{ Meal } & \multicolumn{2}{|c|}{ Pellet } & \multicolumn{2}{|c|}{ Meal } & \multicolumn{2}{|c|}{ Pellet } \\
\hline & $\begin{array}{l}\text { Low } \\
\text { fiber }\end{array}$ & $\begin{array}{l}\text { High } \\
\text { fiber }\end{array}$ & $\begin{array}{l}\text { Low } \\
\text { fiber }\end{array}$ & $\begin{array}{l}\text { High } \\
\text { fiber }\end{array}$ & $\begin{array}{l}\text { Low } \\
\text { fiber }\end{array}$ & $\begin{array}{l}\text { High } \\
\text { fiber }\end{array}$ & $\begin{array}{l}\text { Low } \\
\text { fiber }\end{array}$ & $\begin{array}{l}\text { High } \\
\text { fiber }\end{array}$ \\
\hline$\overline{\mathrm{DM}, \%}$ & 88.54 & 89.24 & 88.91 & 88.42 & 88.58 & 89.17 & 89.14 & 91.40 \\
\hline $\mathrm{CP}, \%$ & 14.9 & 19.4 & 15.0 & 19.5 & 14.0 & 17.8 & 13.4 & 17.7 \\
\hline $\mathrm{ADF}, \%$ & 2.3 & 6.1 & 2.6 & 6.2 & 2.4 & 6.3 & 2.2 & 6.0 \\
\hline NDF, $\%$ & 6.2 & 16.9 & 7.2 & 16.5 & 7.1 & 16.1 & 6.2 & 16.0 \\
\hline $\begin{array}{l}\text { Crude } \\
\text { fiber, \% }\end{array}$ & 2.0 & 4.1 & 1.8 & 4.3 & 1.8 & 4.5 & 1.8 & 4.5 \\
\hline $\begin{array}{l}\text { Ether } \\
\text { extract, } \\
\%\end{array}$ & 1.9 & 4.5 & 2.4 & 3.3 & 2.3 & 4.5 & 2.2 & 5.1 \\
\hline $\mathrm{Ca}, \%$ & 0.45 & 0.46 & 0.43 & 0.57 & 0.30 & 0.47 & 0.40 & 0.54 \\
\hline $\mathrm{P}, \%$ & 0.47 & 0.66 & 0.43 & 0.58 & 0.38 & 0.65 & 0.36 & 0.63 \\
\hline
\end{tabular}

${ }^{1} \mathrm{~A}$ composite sample consisting of 6 subsamples was used for analysis. ${ }^{2}$ Phase 3 diets were fed from d 40 to 64 .

${ }^{3}$ Phase 4 diets were fed from d 64 to 81 .

Growth Performance and Carcass Vield. No diet form $\times$ diet regimen interactions $(P>0.14)$ were observed for growth performance during any of the dietary phases or for the overall trial (Table 7). From d 0 to 64, ADG did not differ among pigs fed different diet forms; however, pigs fed meal diets had increased $(P<0.05)$ ADFI and poorer $(P<0.05) \mathrm{G}: \mathrm{F}$ than pigs fed pelleted diets. Diet type level did not influence ADG, but pigs fed low-fiber, low-fat diets from d 0 to 64 had decreased

Table 6. Analysis of pellet quality, Exp. $1^{1}$

\begin{tabular}{|c|c|c|}
\hline \multirow[b]{2}{*}{ Item } & \multicolumn{2}{|c|}{ Fiber level } \\
\hline & Low $^{2}$ & $\operatorname{High}^{3}$ \\
\hline \multicolumn{3}{|c|}{ Standard pellet durability index, ${ }^{4} \%$} \\
\hline Phase 1 & 91.0 & 92.7 \\
\hline Phase 2 & 90.1 & 96.2 \\
\hline Phase 3 & 92.9 & 95.9 \\
\hline Phase 4 & 94.9 & 91.4 \\
\hline \multicolumn{3}{|c|}{ Modified pellet durability index 5} \\
\hline Phase 1 & 87.9 & 89.4 \\
\hline Phase 2 & 86.3 & 92.7 \\
\hline Phase 3 & 89.5 & 93.8 \\
\hline Phase 4 & 92.4 & 88.8 \\
\hline \multicolumn{3}{|l|}{ Fines, \% } \\
\hline Phase 1 & 7.6 & 7.3 \\
\hline Phase 2 & 9.0 & 7.4 \\
\hline Phase 3 & 8.0 & 8.4 \\
\hline Phase 4 & 7.9 & 8.1 \\
\hline \multicolumn{3}{|c|}{$\begin{array}{l}{ }^{1} \text { A representative feed sample was taken at the feeder during each phase } \\
\text { and analyzed in duplicate for each pellet quality measurement. } \\
{ }^{2} \text { Refers to diet with } 0 \% \text { dried distillers grains with solubles (DDGS) and }\end{array}$} \\
\hline \multicolumn{3}{|c|}{$0 \%$ wheat middlings (midds). } \\
\hline \multicolumn{3}{|c|}{${ }^{3}$ Refers to diet with $30 \%$ DDGS and $19 \%$ midds. } \\
\hline \multicolumn{3}{|c|}{$\begin{array}{l}{ }^{4} \text { Pellet durability index was determined using the standard tumbling- } \\
\text { box technique. }\end{array}$} \\
\hline
\end{tabular}


Table 7. Effects of diet regimen and diet form on finishing pig growth performance, Exp. $1^{1}$

\begin{tabular}{|c|c|c|c|c|c|c|c|c|c|c|}
\hline \multirow[b]{3}{*}{ Item } & \multicolumn{6}{|c|}{ Diet form } & \multirow[b]{3}{*}{ SEM } & \multirow{2}{*}{\multicolumn{3}{|c|}{ Probability $P$}} \\
\hline & \multicolumn{3}{|c|}{$\mathrm{Meal}^{2}$} & \multicolumn{3}{|c|}{ Pellet $^{2}$} & & & & \\
\hline & Low, low & High, low & High, high & Low, low & High, low & High, high & & $\begin{array}{l}\text { Diet form } \\
\times \text { regimen }\end{array}$ & $\begin{array}{c}\text { Meal vs. } \\
\text { pellet }\end{array}$ & $\begin{array}{l}\text { Diet } \\
\text { regimen }\end{array}$ \\
\hline \multicolumn{11}{|l|}{ d 0 to 64} \\
\hline $\mathrm{ADG}, \mathrm{kg}$ & 0.95 & 0.97 & 0.96 & 0.97 & 0.98 & 0.99 & 0.022 & 0.925 & 0.273 & 0.636 \\
\hline ADFI, kg & 2.47 & 2.63 & 2.65 & 2.41 & 2.49 & 2.52 & 0.055 & 0.758 & 0.016 & 0.012 \\
\hline $\mathrm{G}: \mathrm{F}$ & 0.386 & 0.368 & 0.362 & 0.405 & 0.393 & 0.391 & 0.010 & 0.523 & 0.001 & 0.001 \\
\hline \multicolumn{11}{|l|}{ d 64 to 81} \\
\hline $\mathrm{ADG}, \mathrm{kg}$ & 0.93 & 0.97 & 0.88 & 1.02 & 1.03 & 0.97 & 0.032 & 0.886 & 0.005 & 0.026 \\
\hline ADFI, kg & 2.93 & 3.26 & 3.22 & 3.15 & 3.38 & 3.16 & 0.069 & 0.135 & 0.100 & 0.001 \\
\hline G:F & 0.317 & 0.296 & 0.273 & 0.322 & 0.303 & 0.306 & 0.009 & 0.246 & 0.058 & 0.006 \\
\hline \multicolumn{11}{|l|}{ d 0 to 81} \\
\hline $\mathrm{ADG}, \mathrm{kg}$ & 0.95 & 0.97 & 0.94 & 0.98 & 0.99 & 0.98 & 0.017 & 0.829 & 0.029 & 0.354 \\
\hline ADFI, kg & 2.56 & 2.77 & 2.77 & 2.56 & 2.67 & 2.66 & 0.054 & 0.568 & 0.116 & 0.006 \\
\hline $\mathrm{G}: \mathrm{F}$ & 0.369 & 0.350 & 0.341 & 0.384 & 0.370 & 0.370 & 0.005 & 0.192 & 0.001 & 0.001 \\
\hline \multicolumn{11}{|c|}{ Caloric efficiency, kcal/kg gain } \\
\hline ME & 8.97 & 9.32 & 9.66 & 8.63 & 8.83 & 8.88 & 0.121 & 0.193 & 0.001 & 0.002 \\
\hline $\mathrm{NE}$ & 6.82 & 6.89 & 6.98 & 6.56 & 6.54 & 6.42 & 0.088 & 0.218 & 0.001 & 0.965 \\
\hline \multicolumn{11}{|l|}{$\mathrm{BW}, \mathrm{kg}$} \\
\hline $\mathrm{d} 0$ & 49.7 & 49.4 & 49.8 & 49.5 & 49.9 & 49.3 & 1.327 & 0.913 & 0.972 & 0.930 \\
\hline d 64 & 111.0 & 111.4 & 111.2 & 112.5 & 112.7 & 112.4 & 1.869 & 0.996 & 0.371 & 0.882 \\
\hline $\mathrm{d} 81$ & 126.7 & 127.8 & 126.1 & 130.4 & 130.1 & 128.9 & 1.885 & 0.940 & 0.066 & 0.436 \\
\hline Carcass yield, $\%$ & 75.11 & 74.66 & 74.11 & 75.03 & 74.85 & 73.35 & 0.239 & 0.876 & 0.277 & 0.001 \\
\hline $\mathrm{HCW}, \mathrm{kg}$ & 95.3 & 95.4 & 93.5 & 97.9 & 97.5 & 94.7 & 1.314 & 0.131 & 0.080 & 0.105 \\
\hline
\end{tabular}

${ }^{1} \mathrm{~A}$ total of 288 pigs (PIC $327 \times 1050$, initially $49.6 \mathrm{~kg} \mathrm{BW}$ ) were used in an 81-d trial with 6 pens per treatment and 8 pigs per pen.

${ }^{2}$ Low refers to diet with $0 \%$ dried distillers grains with solubles (DDGS) and $0 \%$ wheat middlings (midds); high refers to diet with $30 \%$ DDGS and $19 \%$ midds. The first "high" or "low" designation indicates the amount of fiber fed from d 0 to 64 ; the second indicates the amount of fiber fed from $\mathrm{d} 64$ to 81 .

$(P<0.05)$ ADFI and increased $(P<0.05) \mathrm{G}:$ F compared with pigs fed high-fiber, high-fat diets during this period.

From d 64 to 81, pigs fed pelleted diets had increased $(P<0.05)$ ADG and tended to have increased $(P=0.10)$ ADFI compared with pigs fed meal diets. Feeding pelleted diets also tended to increase $(P<$ $0.10)$ G:F. Pigs previously fed high-fiber, high-fat diets and then switched to low-fiber, low-fat diets during this phase had increased $(P<0.05)$ ADG compared with pigs maintained on the high-fiber, high-fat diets. Pigs fed the low-fiber, low-fat diets throughout the trial had intermediate ADG. Pigs previously fed highfiber, high-fat diets and switched to the low-fiber, lowfat diet had increased $(P<0.05)$ ADFI compared with pigs fed low-fiber, low-fat or high-fiber, high-fat diets throughout the trial. Pigs fed low-fiber, low-fat diets throughout the trial had increased $(P<0.05) \mathrm{G}: \mathrm{F}$ compared with pigs fed high-fiber, high-fat diets throughout, and pigs that were withdrawn from the high-fiber, high-fat diet were intermediate.

Overall (d 0 to 81), there was no difference in ADFI between pigs fed the different diet forms. Pigs fed pelleted diets tended $(P<0.10)$ to have increased final BW and HCW compared with pigs fed meal di- ets, but diet form did not influence carcass yield. Diet regimen did not influence ADG for the overall trial, but pigs fed low-fiber, low-fat diets throughout the trial had decreased $(P<0.05)$ ADFI and improved $(P<0.05) \mathrm{G}: \mathrm{F}$ compared with pigs on the high-fiber, high-fat withdrawal or pigs fed the high-fiber, high-fat regimen throughout. Caloric efficiency was improved $(P<0.05)$ on an ME and NE basis when diets were pelleted. Feeding the high-fiber, high-fat regimen resulted in poorer $(P<0.05)$ caloric efficiency on an ME basis; however, diet regimen did not influence caloric efficiency on a NE basis. Diet regimen did not affect final $\mathrm{BW}$ or $\mathrm{HCW}$, but pigs fed high-fiber, high-fat diets throughout the trial had decreased $(P<0.05)$ carcass yield compared with pigs fed low-fiber, low-fat diets or those withdrawn from high-fiber, high-fat diets on d 64. Removing high-fiber ingredients (DDGS and midds) from the diet before harvest improved carcass yield and returned carcass weights to values similar to that of control pigs fed corn-soybean mealbased diets throughout the trial.

Belly Fatty Acid Composition. Interactive effects between diet form and diet regimen were detected $(P$ $<0.05)$ for palmitic $(\mathrm{C} 16: 0)$ and linoleic $(\mathrm{C} 18: 2 \mathrm{n} 6 \mathrm{c})$ 
Table 8. Effects of diet regimen and diet form on finishing pig belly fatty acid profile, Exp. $1^{1}$

\begin{tabular}{|c|c|c|c|c|c|c|c|c|c|c|}
\hline \multirow[b]{3}{*}{ Item } & \multicolumn{6}{|c|}{ Diet form } & \multirow[b]{3}{*}{ SEM } & \multirow{2}{*}{\multicolumn{3}{|c|}{ Probability $P$}} \\
\hline & \multicolumn{3}{|c|}{$\mathrm{Meal}^{2}$} & \multicolumn{3}{|c|}{ Pellet $^{2}$} & & & & \\
\hline & $\begin{array}{l}\text { Low, } \\
\text { low }\end{array}$ & $\begin{array}{l}\text { High, } \\
\text { low }\end{array}$ & $\begin{array}{l}\text { High, } \\
\text { high }\end{array}$ & $\begin{array}{l}\text { Low, } \\
\text { low }\end{array}$ & $\begin{array}{l}\text { High, } \\
\text { low }\end{array}$ & $\begin{array}{l}\text { High, } \\
\text { high }\end{array}$ & & $\begin{array}{l}\text { Diet form } \\
\times \text { regimen }\end{array}$ & $\begin{array}{c}\text { Meal vs. } \\
\text { pellet }\end{array}$ & $\begin{array}{l}\text { Diet } \\
\text { regimen }\end{array}$ \\
\hline Myristic acid (C14:0), \% & 1.47 & 1.39 & 1.36 & 1.44 & 1.31 & 1.29 & 0.018 & 0.588 & 0.001 & 0.001 \\
\hline Palmitic acid (C16:0), \% & 23.91 & 22.49 & 21.87 & 23.68 & 21.67 & 21.04 & 0.130 & 0.049 & 0.001 & 0.001 \\
\hline Palmitoleic acid (C16:1), \% & 3.30 & 3.06 & 2.96 & 3.03 & 2.66 & 2.62 & 0.061 & 0.805 & 0.001 & 0.001 \\
\hline Margaric acid (C17:0), \% & 0.35 & 0.39 & 0.43 & 0.33 & 0.36 & 0.38 & 0.014 & 0.449 & 0.002 & 0.001 \\
\hline Stearic acid (C18:0), \% & 10.61 & 9.44 & 8.94 & 10.79 & 9.21 & 8.64 & 0.114 & 0.073 & 0.187 & 0.001 \\
\hline Oleic acid $(\mathrm{C} 18: \ln 9 \mathrm{c}), \%$ & 39.45 & 37.84 & 36.73 & 38.71 & 36.59 & 35.73 & 0.214 & 0.652 & 0.001 & 0.001 \\
\hline Vaccenic acid (C18:1n7), \% & 4.27 & 3.95 & 3.76 & 4.02 & 3.57 & 3.47 & 0.051 & 0.865 & 0.001 & 0.001 \\
\hline Linoleic acid (C18:2n6c), \% & 12.89 & 17.22 & 19.57 & 14.25 & 20.38 & 22.51 & 0.290 & 0.012 & 0.001 & 0.001 \\
\hline Total C18:2 fatty acids, ${ }^{3} \%$ & 13.05 & 17.41 & 19.75 & 14.38 & 20.52 & 22.64 & 0.290 & 0.012 & 0.001 & 0.001 \\
\hline$\alpha$-Linolenic acid (C18:3n3), \% & 0.58 & 0.68 & 0.74 & 0.63 & 0.80 & 0.84 & 0.014 & 0.163 & 0.001 & 0.001 \\
\hline Arachidic acid (C20:0), \% & 0.22 & 0.22 & 0.21 & 0.23 & 0.22 & 0.21 & 0.004 & 0.535 & 0.572 & 0.001 \\
\hline Eicosenoic acid (C20:1), \% & 0.65 & 0.67 & 0.66 & 0.67 & 0.66 & 0.63 & 0.015 & 0.333 & 0.580 & 0.364 \\
\hline Eicosadienoic acid (C20:2), \% & 0.59 & 0.78 & 0.85 & 0.65 & 0.90 & 0.95 & 0.012 & 0.153 & 0.001 & 0.001 \\
\hline Arachidonic acid (C20:4n6), \% & 0.25 & 0.29 & 0.30 & 0.24 & 0.28 & 0.29 & 0.006 & 0.839 & 0.154 & 0.001 \\
\hline Other fatty acids, $\%$ & 1.30 & 1.42 & 1.46 & 1.22 & 1.26 & 1.29 & 0.018 & 0.049 & 0.001 & 0.001 \\
\hline Total SFA, ${ }^{4} \%$ & 36.94 & 34.29 & 33.18 & 36.82 & 33.12 & 31.90 & 0.208 & 0.011 & 0.001 & 0.001 \\
\hline Total MUFA, ${ }^{5} \%$ & 48.25 & 46.16 & 44.76 & 46.95 & 43.99 & 42.96 & 0.286 & 0.559 & 0.001 & 0.001 \\
\hline Total PUFA, ${ }^{6} \%$ & 14.80 & 19.55 & 22.06 & 16.23 & 22.89 & 25.15 & 0.318 & 0.018 & 0.001 & 0.001 \\
\hline UFA:SFA ${ }^{7}$ & 1.71 & 1.92 & 2.02 & 1.72 & 2.03 & 2.14 & 0.018 & 0.005 & 0.001 & 0.001 \\
\hline PUFA:SFA ${ }^{8}$ & 0.40 & 0.57 & 0.67 & 0.44 & 0.69 & 0.79 & 0.012 & 0.001 & 0.001 & 0.001 \\
\hline Iodine value, ${ }^{9} \mathrm{mg} / \mathrm{g}$ & 65.7 & 71.7 & 74.7 & 67.0 & 75.5 & 78.4 & 0.378 & 0.003 & 0.001 & 0.001 \\
\hline
\end{tabular}

${ }^{1}$ All items calculated as a percentage of the total fatty acid content. Belly fat samples were collected from the ventral side of the belly along the navel edge between the 10th and the 12th ribs.

${ }^{2}$ Low refers to diet with $0 \%$ dried distillers grains with solubles (DDGS) and $0 \%$ wheat middlings (midds); high refers to diet with 30\% DDGS and 19\% midds. The first "high" or "low" designation indicates the amount of fiber fed from d 0 to 64 ; the second indicates the amount of fiber fed from $\mathrm{d} 64$ to 81 .

${ }^{3}$ Total $\mathrm{C} 18: 2$ fatty acids $=[\% \mathrm{C} 18: 2 \mathrm{n} 6 \mathrm{t}]+[\% \mathrm{C} 18: 2 \mathrm{n} 6 \mathrm{c}]+[\% \mathrm{C} 18: 2,9 \mathrm{c} 11 \mathrm{t}]+[\% 18: 2,10 \mathrm{t} 12 \mathrm{c}]+[\% \mathrm{C} 18: 2,9 \mathrm{c} 11 \mathrm{c}]+[\mathrm{C} 18: 2,9 \mathrm{t} 11 \mathrm{t}]$.

${ }^{4}$ Total SFA $=[\% \mathrm{C} 10: 0]+[\% \mathrm{C} 11: 0]+[\% \mathrm{C} 12: 0]+[\% \mathrm{C} 14: 0]+[\% \mathrm{C} 15: 0]+[\% \mathrm{C} 16: 0]+[\% \mathrm{C} 17: 0]+[\% \mathrm{C} 18: 0]+[\% \mathrm{C} 20: 0]+[\% \mathrm{C} 21: 0]+[\%$ $\mathrm{C} 22: 0]+[\% \mathrm{C} 24: 0]$.

${ }^{5}$ Total MUFA $=[\% \mathrm{C} 14: 1]+[\% \mathrm{C} 15: 1]+[\% \mathrm{C} 16: 1]+[\% \mathrm{C} 17: 1]+[\% \mathrm{C} 18: \ln 9 \mathrm{t}]+[\% \mathrm{C} 18: 1 \mathrm{n} 9 \mathrm{c}]+[\% \mathrm{C} 18: 1 \mathrm{n} 7]+[\% \mathrm{C} 20: 1]+[\% \mathrm{C} 24: 1]$.

${ }^{6}$ Total PUFA $=[\% \mathrm{C} 18: 2 \mathrm{n} 6 \mathrm{t}]+[\% \mathrm{C} 18: 2 \mathrm{n} 6 \mathrm{c}]+[\% \mathrm{C} 18: 29 \mathrm{c}, 11 \mathrm{t}]+[\% \mathrm{C} 18: 210 \mathrm{t}, 12 \mathrm{c}]+[\% \mathrm{C} 18: 29 \mathrm{c}, 11 \mathrm{c}]+[\% \mathrm{C} 18: 29 \mathrm{t}, 11 \mathrm{t}]+[\% \mathrm{C} 18: 3 \mathrm{n} 6]+[\%$ $\mathrm{C} 18: 3 \mathrm{n} 3]+[\% \mathrm{C} 20: 2]+[\% \mathrm{C} 20: 3 \mathrm{n} 6]+[\% \mathrm{C} 20: 4 \mathrm{n} 6]+[\% \mathrm{C} 20: 5 \mathrm{n} 3]+[\% \mathrm{C} 22: 5 \mathrm{n} 3]+[\% \mathrm{C} 22: 5 \mathrm{n} 6]$

${ }^{7}$ UFA $:$ SFA ratio $=[$ total MUFA + total PUFA $] /$ total SFA.

${ }^{8} \mathrm{PUFA}: \mathrm{SFA}$ ratio $=$ total PUFA/total SFA.

${ }^{9}$ Iodine value $=[\% \mathrm{C} 16: 1] \times 0.95+[\% \mathrm{C} 18: 1] \times 0.86+[\% \mathrm{C} 18: 2] \times 1.732+[\% \mathrm{C} 18: 3] \times 2.616+[\% \mathrm{C} 20: 1] \times 0.785+[\% \mathrm{C} 22: 1] \times 0.723$.

acid concentrations (Table 8). Pelleting decreased palmitic and increased linoleic acid by a greater magnitude when the diet contained high fiber and fat contents than when the diet was low in fiber and fat. Palmitic and total C18:2 fatty acids account for the greatest portions of SFA and PUFA, respectively. As a result, there were also interactions $(P<0.05)$ for belly fat IV, total SFA, total PUFA, UFA:SFA ratio, PUFA:SFA ratio, and belly fat IV. Pelleting the diets also increased belly fat IV and UFA, with the magnitude of response being greater when the high-fiber diets were pelleted compared with the low-fiber diets. On average, pelleting the diets increased belly fat IV by $2.9 \mathrm{mg} / \mathrm{g}$.

Feeding pelleted diets reduced $(P<0.05)$ myristic (C14:0), palmitic (C16:0), palmitoleic (C16:1), marga- ric $(\mathrm{C} 17: 0)$, oleic $(\mathrm{C} 18: 1 \mathrm{n} 9 \mathrm{c})$, and vaccenic $(\mathrm{C} 18: 1 \mathrm{n} 7)$ fatty acids; however, it increased $(P<0.05)$ linoleic (C18:2n6c), $\alpha$-linolenic (C18:3n3), eicosadienoic (C20:2), and total C18:2 fatty acids ( 8). As a result, total PUFA and belly fat IV increased $(P<0.05)$, whereas total SFA, MUFA, and all other fatty acids decreased $(P$ $<0.05)$ when pigs were fed pelleted diets. There were no differences $(P>0.10)$ in stearic $(\mathrm{C} 18: 0)$, arachidic (20:0), eicosenoic (20:1), and arachidonic (C20:4n6) fatty acids between pigs fed the different diet forms.

Compared with pigs fed high-fat, high-fiber diets throughout the trial, pigs fed low-fiber, low-fat regimens throughout the trial had increased $(P<0.05)$ $\mathrm{C} 16: 0, \mathrm{C} 18: 0, \mathrm{C} 18: 1 \mathrm{n} 9 \mathrm{c}, \mathrm{C} 18: 1 \mathrm{n} 7$, total SFA, and total MUFA concentrations, with those fed the withdrawal 
Table 9. Chemical analysis of diets, Exp. $2^{1}$

\begin{tabular}{|c|c|c|c|c|c|c|c|c|c|c|c|c|}
\hline \multirow[b]{3}{*}{ Item } & \multicolumn{6}{|c|}{ Phase $1^{2}$} & \multicolumn{6}{|c|}{ Phase $2^{3}$} \\
\hline & \multicolumn{3}{|c|}{ Meal } & \multicolumn{3}{|c|}{ Pellet } & \multicolumn{3}{|c|}{ Meal } & \multicolumn{3}{|c|}{ Pellet } \\
\hline & Control & $\begin{array}{c}\text { DDGS + } \\
\text { midds }\end{array}$ & $\begin{array}{c}\text { Corn } \\
\text { oil }\end{array}$ & Control & $\begin{array}{c}\text { DDGS + } \\
\text { Midds }\end{array}$ & $\begin{array}{c}\text { Corn } \\
\text { oil }\end{array}$ & Control & $\begin{array}{c}\text { DDGS + } \\
\text { midds }\end{array}$ & $\begin{array}{c}\text { Corn } \\
\text { oil }\end{array}$ & Control & $\begin{array}{c}\text { DDGS + } \\
\text { midds }\end{array}$ & $\begin{array}{c}\text { Corn } \\
\text { oil }\end{array}$ \\
\hline $\mathrm{DM}, \%$ & 89.47 & 90.73 & 89.62 & 89.18 & 88.94 & 89.33 & 89.60 & 90.63 & 89.47 & 89.46 & 88.64 & 89.13 \\
\hline $\mathrm{CP}, \%$ & 17.7 & 18.4 & 18.0 & 18.8 & 23.0 & 18.1 & 18.1 & 18.6 & 17.4 & 16.9 & 21.6 & 17.0 \\
\hline $\mathrm{ADF}, \%$ & 2.7 & 7.1 & 2.6 & 3 & 7.3 & 3.1 & 3.2 & 7.3 & 3.3 & 2.9 & 6.4 & 3.2 \\
\hline NDF, \% & 7.6 & 17.7 & 6.6 & 6.7 & 16.6 & 6.6 & 5.9 & 18.0 & 6.7 & 5.1 & 14.0 & 5.3 \\
\hline Crude fiber, \% & 2.0 & 4.7 & 2.0 & 1.8 & 4.5 & 2.0 & 2.1 & 4.8 & 2.1 & 1.8 & 4.0 & 2.0 \\
\hline Ether extract, \% & 1.6 & 4.3 & 3.5 & 1.4 & 3.4 & 3.7 & 1.5 & 4.3 & 3.7 & 1.5 & 3.2 & 3.1 \\
\hline $\mathrm{Ca}, \%$ & 0.98 & 0.45 & 0.83 & 0.58 & 0.62 & 0.64 & 0.62 & 0.58 & 0.54 & 0.65 & 0.60 & 0.53 \\
\hline P, \% & 0.49 & 0.65 & 0.51 & 0.48 & 0.66 & 0.49 & 0.46 & 0.66 & 0.44 & 0.44 & 0.62 & 0.42 \\
\hline
\end{tabular}

${ }^{1}$ A composite sample consisting of 6 subsamples was used for analysis. DDGS = dried distillers grains with solubles; midds = wheat middlings.

${ }^{2}$ Phase 1 diets were fed from d 0 to 21.

${ }^{3}$ Phase 2 diets were fed from d 21 to 45 .

regimen intermediate $(P<0.05)$. Pigs fed the low-fiber, low-fat diet had decreased $(P<0.05) \mathrm{C} 18: 2 \mathrm{n} 6 \mathrm{C}$, $\mathrm{C} 18: 3 \mathrm{n} 3, \mathrm{C} 20: 2, \mathrm{C} 20: 4 \mathrm{n} 6$, total C18:2, PUFA, and belly fat IV compared with those fed high-fiber, highfat diets, with those on the withdrawal regimen intermediate $(P<0.05)$.

Regardless of withdrawal, pigs fed high-fiber, high-fat diets during any period of the experiment had decreased $(P<0.05) \mathrm{C} 14: 0$ and $\mathrm{C} 16: 1$ concentrations and increased $(P<0.05) \mathrm{C} 17: 0$ concentrations compared with pigs fed low-fiber, low-fat diets for the entire trial. Feeding high-fiber, high-fat diets throughout the experiment decreased $(P<0.05)$ C20:0 concentrations compared with the other 2 regimens. No differences were detected in C20:1 among pigs fed the different diet regimens.

\section{Experiment 2}

Chemical Analysis and Pellet Quality Measurements. Analysis of diets revealed that as expected, the inclusion of dietary DDGS and midds increased ADF, NDF, crude fiber, and ether extract (Tables 9 and 10). In addition, ether extract increased because of the inclusion of corn oil, which was also expected. Standard PDI was greater than $88 \%$ during all phases for pelleted diets, with modified PDI ranging from $82.5 \%$ to $87.5 \%$ (Table 11). Percentage of fines was low for all diets and phases, ranging from approximately $7 \%$ to $14 \%$ fines.

Growth Performance and Carcass Yield. No diet form $\times$ diet type interactions were observed for growth performance, HCW, or carcass yield for the overall trial (Table 12). Overall (d 0 to 87), pigs fed pelleted diets had increased $(P<0.05)$ ADG, decreased $(P<$ $0.05)$ ADFI, and improved $(P<0.05) \mathrm{G}: \mathrm{F}$ and caloric efficiency compared with pigs fed meal diets (12). Pigs fed pelleted diets tended $(P<0.10)$ to have in-

Table 10. Chemical analysis of diets, Exp. $2^{1}$

\begin{tabular}{|c|c|c|c|c|c|c|c|c|c|c|c|c|}
\hline \multirow[b]{3}{*}{ Item } & \multicolumn{6}{|c|}{ Phase $3^{2}$} & \multicolumn{6}{|c|}{ Phase $4^{3}$} \\
\hline & \multicolumn{3}{|c|}{ Meal } & \multicolumn{3}{|c|}{ Pellet } & \multicolumn{3}{|c|}{ Meal } & \multicolumn{3}{|c|}{ Pellet } \\
\hline & Control & $\begin{array}{c}\text { DDGS + } \\
\text { midds }\end{array}$ & $\begin{array}{c}\text { Corn } \\
\text { oil }\end{array}$ & Control & $\begin{array}{c}\text { DDGS + } \\
\text { midds }\end{array}$ & $\begin{array}{c}\text { Corn } \\
\text { oil }\end{array}$ & Control & $\begin{array}{c}\text { DDGS + } \\
\text { midds }\end{array}$ & $\begin{array}{c}\text { Corn } \\
\text { oil }\end{array}$ & Control & $\begin{array}{c}\text { DDGS + } \\
\text { midds }\end{array}$ & $\begin{array}{c}\text { Corn } \\
\text { oil }\end{array}$ \\
\hline$\overline{\mathrm{DM}, \%}$ & 89.09 & 90.58 & 89.41 & 88.77 & 90.16 & 88.93 & 89.85 & 90.15 & 89.81 & 89.74 & 91.89 & 90.61 \\
\hline $\mathrm{CP}, \%$ & 17.1 & 21 & 15.3 & 16.3 & 20.8 & 15.9 & 14.2 & 22.3 & 14.0 & 14.5 & 18.4 & 14.3 \\
\hline $\mathrm{ADF}, \%$ & 2.8 & 7.6 & 3.0 & 3.4 & 5.9 & 2.8 & 3.4 & 8.2 & 2.8 & 3.3 & 6.5 & 2.6 \\
\hline NDF, $\%$ & 6.4 & 16 & 6.4 & 5.9 & 14.4 & 6.6 & 6.7 & 16.8 & 6.3 & 5.1 & 16.5 & 5.1 \\
\hline Crude fiber, \% & 1.7 & 4.2 & 2.0 & 1.8 & 3.5 & 1.9 & 2.1 & 4.8 & 2.1 & 1.6 & 4.3 & 1.5 \\
\hline Ether extract, \% & 2.0 & 4.6 & 4.2 & 1.6 & 4.4 & 3.8 & 2.0 & 3.7 & 3.2 & 1.9 & 4.7 & 3.5 \\
\hline $\mathrm{Ca}, \%$ & 0.38 & 0.47 & 0.61 & 0.45 & 0.61 & 0.55 & 0.90 & 0.88 & 1.19 & 0.98 & 0.56 & 0.56 \\
\hline $\mathrm{P}, \%$ & 0.49 & 0.66 & 0.49 & 0.46 & 0.66 & 0.44 & 0.47 & 0.58 & 0.51 & 0.44 & 0.64 & 0.40 \\
\hline
\end{tabular}

${ }^{1} \mathrm{~A}$ composite sample consisting of 6 subsamples was used for analysis. DDGS = dried distillers grains with solubles; midds = wheat middlings.

${ }^{2}$ Phase 3 diets were fed from $\mathrm{d} 45$ to 70 .

${ }^{3}$ Phase 4 diets were fed from d 70 to 87. 
Table 12. Effects of diet form and type on growth performance and carcass yield, Exp. $2^{1}$

\begin{tabular}{|c|c|c|c|c|c|c|c|c|c|c|}
\hline \multirow[b]{3}{*}{ Item } & \multicolumn{6}{|c|}{ Diet form } & \multirow[b]{3}{*}{ SEM } & \multirow{2}{*}{\multicolumn{3}{|c|}{ Probability $P$}} \\
\hline & \multicolumn{3}{|c|}{ Meal } & \multicolumn{3}{|c|}{ Pellet } & & & & \\
\hline & Control $^{2}$ & $\begin{array}{c}\text { DDGS + } \\
\text { midds }^{3}\end{array}$ & Corn oil ${ }^{4}$ & Control $^{2}$ & $\begin{array}{c}\text { DDGS + } \\
\text { midds }^{3}\end{array}$ & Corn oil ${ }^{4}$ & & $\begin{array}{l}\text { Diet form } \\
\times \text { type }\end{array}$ & Diet form & Diet type \\
\hline Initial BW, kg & 48.5 & 48.5 & 48.5 & 48.5 & 48.5 & 48.5 & 1.041 & 0.998 & 0.997 & 0.996 \\
\hline \multicolumn{11}{|l|}{ d 0 to 87} \\
\hline $\mathrm{ADG}, \mathrm{kg}$ & 0.95 & 0.91 & 0.95 & 0.96 & 0.94 & 0.98 & 0.013 & 0.706 & 0.038 & 0.009 \\
\hline ADFI, $\mathrm{kg}$ & 2.64 & 2.66 & 2.52 & 2.49 & 2.63 & 2.44 & 0.042 & 0.372 & 0.016 & 0.002 \\
\hline $\mathrm{G}: \mathrm{F}$ & 0.359 & 0.341 & 0.378 & 0.385 & 0.357 & 0.401 & 0.005 & 0.619 & 0.001 & 0.001 \\
\hline \multicolumn{11}{|c|}{ Caloric efficiency, Mcal/kg gain } \\
\hline ME & 9.24 & 9.93 & 9.19 & 8.61 & 9.09 & 8.64 & 0.125 & 0.744 & 0.001 & 0.005 \\
\hline $\mathrm{NE}$ & 7.00 & 6.94 & 7.04 & 6.53 & 6.62 & 6.62 & 0.094 & 0.699 & 0.001 & 0.699 \\
\hline Final BW, kg & 130.8 & 127.3 & 131.4 & 131.7 & 130.1 & 134.5 & 1.521 & 0.747 & 0.076 & 0.028 \\
\hline $\mathrm{HCW}, \mathrm{kg}$ & 98.6 & 93.9 & 98.6 & 98.2 & 95.9 & 101.6 & 1.218 & 0.366 & 0.132 & 0.001 \\
\hline Carcass yield, $\%$ & 75.37 & 73.82 & 75.01 & 74.53 & 73.75 & 75.54 & 0.309 & 0.163 & 0.619 & 0.001 \\
\hline
\end{tabular}

An interactive effect between diet form and oil source was detected $(P<0.05)$ for shoulder fat IV, which resulted from pigs fed the control or corn oil diet having higher shoulder fat IV when diets were fed as pellets compared with meal form; however, pigs fed the DDGS and midds diet had a slight numeric decrease in shoulder fat IV when fed pelleted diets compared with meal.

\section{DISCUSSION}

In Exp. 1, feeding diets containing 30\% DDGS and $19 \%$ midds did not influence ADG from d 0 to 64 , but when pigs were withdrawn from the high-fiber, highfat diet and switched to a corn-soybean meal-based diet from d 64 to 81, ADG increased compared with that of pigs that remained on the high-fiber, high-fat diet throughout. This response was driven primarily by increases in ADFI. The diets fed in Exp. 1 were similar to those used by Asmus et al. (2014), who investigated the effects of reducing or completely removing DDGS and midds from finishing pig diets for multiple durations. The authors reported similar increases in ADG and ADFI when the high-fiber components were withdrawn from the diet and attributed this effect to the bulk density and energy content of the diets; that is, pigs previously fed high-fiber diets continued to consume higher volumetric amounts of feed despite switching to a more energy-dense diet. Overall, for both experiments, pigs fed the control diets without DDGS or midds had similar ADG and improved G:F compared with those fed 30\% DDGS and 19\% midds for the entirety of each trial. This is in agreement with the results of Salyer et al. (2012), who reported that pigs fed the combination of DDGS and midds had poorer feed efficiency than those fed diets without cereal grain byproducts. The response to feed efficiency was a result of the energy content in the diets, which was further demonstrated by pigs having similar caloric efficiency on a NE basis in both experiments. The fact that caloric efficiency was influenced by diet type on an ME basis but not on a NE basis illustrates that NE more appropriately describes the energy differences between the diets in these experiments.

Feeding pelleted diets improved growth performance compared with feeding meal diets in both experiments. The overall improvements in ADG were $4 \%$ and 3\% for Exp. 1 and 2, respectively, whereas the improvements in G:F were approximately $6 \%$ for both experiments. These results are in agreement with those of Wondra et al. (1995), who observed a $4 \%$ to $6 \%$ increase in $\mathrm{ADG}$ and $7 \%$ improvement in G:F when feeding pelleted diets compared with meal. De Jong et al. (2013a,b) also reported improvements of approximately $6 \%$ for ADG and G:F when diets were fed in pellet instead of meal form. However, the beneficial effects of pelleting are not consistent among all research. Myers et al. (2013) found no benefit in G:F when feeding pelleted diets compared with meal. The authors suggested that the lack of response could be partially due to poor pellet quality and a high percentage of fines present in the pelleted diet, leading to increased feed wastage. This is in agreement with the results of Stark et al. (1993) and Nemechek et al. (2012), who reported that feed efficiency of finishing pigs worsened as the percentage of fines in pelleted 
Table 13. Effects of diet form and type on belly fatty acid profile, Exp. $2^{1}$

\begin{tabular}{|c|c|c|c|c|c|c|c|c|c|c|}
\hline \multirow[b]{3}{*}{ Item } & \multicolumn{6}{|c|}{ Diet form } & \multirow[b]{3}{*}{ SEM } & \multirow{2}{*}{\multicolumn{3}{|c|}{ Probability $P$}} \\
\hline & \multicolumn{3}{|c|}{ Meal } & \multicolumn{3}{|c|}{ Pellet } & & & & \\
\hline & Control $^{2}$ & $\begin{array}{c}\text { DDGS + } \\
\text { midds }^{3}\end{array}$ & $\begin{array}{c}\text { Corn } \\
\text { oil }^{4}\end{array}$ & Control $^{2}$ & $\begin{array}{c}\text { DDGS + } \\
\text { midds }^{3}\end{array}$ & $\begin{array}{c}\text { Corn } \\
\text { oil }^{4}\end{array}$ & & $\begin{array}{l}\text { Diet form } \\
\times \text { type }\end{array}$ & $\begin{array}{c}\text { Meal vs. } \\
\text { pellet }\end{array}$ & Diet type \\
\hline Myristic acid (C14:0), \% & 1.39 & 1.39 & 1.36 & 1.41 & 1.30 & 1.33 & 0.020 & 0.028 & 0.034 & 0.022 \\
\hline Palmitic acid (C16:0), \% & 23.62 & 22.16 & 22.48 & 23.20 & 22.27 & 22.27 & 0.152 & 0.239 & 0.177 & 0.001 \\
\hline Palmitoleic acid (C16:1), \% & 3.29 & 2.95 & 2.65 & 2.76 & 2.46 & 2.47 & 0.076 & 0.056 & 0.001 & 0.001 \\
\hline Margaric acid (C17:0), \% & 0.24 & 0.27 & 0.21 & 0.22 & 0.27 & 0.19 & 0.016 & 0.545 & 0.239 & 0.001 \\
\hline Stearic acid (C18:0), \% & 10.44 & 9.07 & 9.65 & 10.91 & 9.24 & 9.66 & 0.116 & 0.148 & 0.032 & 0.001 \\
\hline Oleic acid $(\mathrm{C} 18: \ln 9 \mathrm{c}), \%$ & 46.66 & 42.56 & 42.55 & 44.99 & 41.66 & 42.31 & 0.239 & 0.019 & 0.001 & 0.001 \\
\hline Vaccenic acid (C18:1n7), \% & 0.93 & 0.84 & 0.63 & 0.73 & 0.64 & 0.61 & 0.048 & 0.130 & 0.001 & 0.001 \\
\hline Total C18:1 fatty acids, $5 \%$ & 47.61 & 43.40 & 43.11 & 45.69 & 42.25 & 42.88 & 0.265 & 0.013 & 0.001 & 0.001 \\
\hline Linoleic acid (C18:2n6c), \% & 11.31 & 18.30 & 18.15 & 13.62 & 19.64 & 18.87 & 0.268 & 0.020 & 0.001 & 0.001 \\
\hline Total C18:2 fatty acids, ${ }^{6} \%$ & 11.94 & 19.13 & 18.93 & 14.31 & 20.53 & 19.68 & 0.274 & 0.021 & 0.001 & 0.001 \\
\hline$\alpha$-Linolenic acid (C18:3n3), \% & 0.70 & 0.72 & 0.65 & 0.66 & 0.69 & 0.64 & 0.022 & 0.765 & 0.171 & 0.037 \\
\hline Eicosenoic acid (C20:1), \% & 0.43 & 0.71 & 0.69 & 0.53 & 0.77 & 0.73 & 0.016 & 0.181 & 0.001 & 0.001 \\
\hline Total SFA, ${ }^{7} \%$ & 35.69 & 32.89 & 33.70 & 35.74 & 33.07 & 33.45 & 0.202 & 0.545 & 0.958 & 0.001 \\
\hline Total MUFA, ${ }^{8} \%$ & 51.33 & 47.05 & 46.45 & 48.99 & 45.48 & 46.08 & 0.318 & 0.014 & 0.001 & 0.001 \\
\hline Total PUFA, ${ }^{9} \%$ & 12.60 & 19.77 & 19.49 & 14.92 & 21.16 & 20.23 & 0.263 & 0.019 & 0.001 & 0.001 \\
\hline UFA:SFA ${ }^{10}$ & 1.79 & 2.03 & 1.96 & 1.79 & 2.02 & 1.98 & 0.017 & 0.486 & 0.885 & 0.001 \\
\hline PUFA:SFA ${ }^{11}$ & 0.35 & 0.60 & 0.58 & 0.42 & 0.64 & 0.61 & 0.009 & 0.096 & 0.001 & 0.001 \\
\hline Iodine value, ${ }^{12} \mathrm{mg} / \mathrm{g}$ & 66.74 & 75.40 & 74.31 & 68.65 & 76.45 & 75.26 & 0.300 & 0.229 & 0.001 & 0.001 \\
\hline
\end{tabular}

${ }^{1}$ All items calculated as a percentage of the total fatty acid content. Belly fat samples were collected from the ventral side of the belly along the navel edge between the 10th and the 12 th ribs.

${ }^{2}$ Corn-soybean meal-based control diet with $0 \%$ dried distillers grains with solubles (DDGS), $0 \%$ wheat middlings (midds), and $0 \%$ corn oil.

${ }^{3}$ Control diet with $30 \%$ DDGS and $19 \%$ midds.

${ }^{4}$ Control diet with $3 \%$ corn oil.

${ }^{5}$ Total $\mathrm{C} 18: 1$ fatty acids $=[\% \mathrm{C} 18: \ln 9 \mathrm{c}]+[\% \mathrm{C} 18: 1 \mathrm{n} 7]$.

${ }^{6}$ Total $\mathrm{C} 18: 2$ fatty acids $=[\% \mathrm{C} 18: 2 \mathrm{n} 6 \mathrm{t}]+[\% \mathrm{C} 18: 2 \mathrm{n} 6 \mathrm{c}]+[\% \mathrm{C} 18: 2,9 \mathrm{c} 11 \mathrm{t}]+[\% 18: 2,10 \mathrm{t} 12 \mathrm{c}]+[\% \mathrm{C} 18: 2,9 \mathrm{c} 11 \mathrm{c}]+[\mathrm{C} 18: 2,9 \mathrm{t} 11 \mathrm{t}]$.

${ }^{7} \mathrm{Total} \mathrm{SFA}=[\% \mathrm{C} 14: 0]+[\% \mathrm{C} 15: 0]+[\% \mathrm{C} 16: 0]+[\% \mathrm{C} 17: 0]+[\% \mathrm{C} 18: 0]+[\% \mathrm{C} 20: 0]+[\% \mathrm{C} 21: 0]+[\% \mathrm{C} 22: 0]+[\% \mathrm{C} 24: 0]$.

${ }^{8}$ Total MUFA $=[\% \mathrm{C} 14: 1]+[\% \mathrm{C} 15: 1]+[\% \mathrm{C} 16: 1]+[\% \mathrm{C} 17: 1]+[\% \mathrm{C} 18: \ln 9 \mathrm{t}]+[\% \mathrm{C} 18: \ln 9 \mathrm{c}]+[\% \mathrm{C} 18: 1 \mathrm{n} 7]+[\% \mathrm{C} 20: 1]+[\% \mathrm{C} 24: 1]$.

${ }^{9}$ Total PUFA $=[\% \mathrm{C} 18: 2 \mathrm{n} 6 \mathrm{t}]+[\% \mathrm{C} 18: 2 \mathrm{n} 6 \mathrm{c}]+[\% \mathrm{C} 18: 29 \mathrm{c}, 11 \mathrm{t}]+[\% \mathrm{C} 18: 210 \mathrm{t}, 12 \mathrm{c}]+[\% \mathrm{C} 18: 29 \mathrm{c}, 11 \mathrm{c}]+[\% \mathrm{C} 18: 29 \mathrm{t}, 11 \mathrm{t}]+[\% \mathrm{C} 18: 3 \mathrm{n} 6]+[\%$ C18:3n3].

${ }^{10}$ UFA:SFA ratio $=[$ total MUFA + total PUFA $] /$ total SFA

${ }^{11}$ PUFA:SFA ratio $=$ total PUFA/total SFA.

${ }^{12}$ Iodine value $=[\% \mathrm{C} 16: 1] \times 0.95+[\% \mathrm{C} 18: 1] \times 0.86+[\% \mathrm{C} 18: 2] \times 1.732+[\% \mathrm{C} 18: 3] \times 2.616+[\% \mathrm{C} 20: 1] \times 0.785$.

diets increased to above $20 \%$. Our research further supports this concept because growth benefits were observed in the current trials when pigs were fed pelleted diets with high PDI and low percentages of fines.

As in previously reported data (Linneen et al., 2008; Salyer et al., 2012), feeding DDGS and midds before harvest resulted in reduced carcass yield in both experiments. In Exp. 1, however, withdrawing these ingredients $17 \mathrm{~d}$ before market improved carcass yield and returned carcass weights to values similar to those of the control-fed pigs. Asmus et al. (2014) also demonstrated that switching pigs from a high-fiber diet to a low-fiber diet $23 \mathrm{~d}$ before market allowed for full recovery of carcass yield. In agreement, Coble et al. (2014) reported that removing DDGS and midds 15 to $20 \mathrm{~d}$ before harvest allowed carcass yield to return to levels of pigs fed corn-soybean meal-based di- ets throughout the finishing period. Because the fiber component of the diets caused reduced carcass yield, it was expected that pigs fed the diet containing 3\% corn oil in Exp. 2 had carcass yield similar to that of the control-fed pigs. Diet form did not influence carcass yield in either experiment, which agrees with the results of Wondra et al. (1995) and Myers et al. (2013), who reported no differences in carcass yield when finishing pigs were fed diets in meal or pelleted form.

As expected, feeding ingredients high in UFA increased carcass fat IV, which agrees with numerous other publications (Benz et al., 2010, 2011; Cromwell et al., 2011). In Exp. 1, pigs fed the high-fiber, highfat diet to market had increased concentrations of total C18:2 and PUFA in belly adipose tissue compared with those fed low-fiber, low-fat diets throughout the trial, with those on the withdrawal regimen interme- 
Table 14. Effects of diet form and type on shoulder fatty acid profile, Exp. $2^{1}$

\begin{tabular}{|c|c|c|c|c|c|c|c|c|c|c|}
\hline \multirow[b]{3}{*}{ Item } & \multicolumn{6}{|c|}{ Diet form } & \multirow[b]{3}{*}{ SEM } & \multirow{2}{*}{\multicolumn{3}{|c|}{ Probability $P$}} \\
\hline & \multicolumn{3}{|c|}{ Meal } & \multicolumn{3}{|c|}{ Pellet } & & & & \\
\hline & Control $^{2}$ & $\begin{array}{c}\text { DDGS + } \\
\text { midds }^{3}\end{array}$ & $\begin{array}{c}\text { Corn } \\
\text { oil }^{4}\end{array}$ & Control $^{2}$ & $\begin{array}{c}\text { DDGS + } \\
\text { midds }^{3}\end{array}$ & $\begin{array}{c}\text { Corn } \\
\text { oil }^{4}\end{array}$ & & $\begin{array}{l}\text { Diet form } \times \\
\text { type }\end{array}$ & $\begin{array}{c}\text { Meal vs. } \\
\text { pellet }\end{array}$ & Diet type \\
\hline Myristic acid (C14:0), \% & 1.35 & 1.28 & 1.25 & 1.29 & 1.22 & 1.19 & 0.021 & 0.956 & 0.002 & 0.001 \\
\hline Palmitic acid (C16:0), \% & 23.82 & 22.46 & 22.54 & 22.96 & 23.23 & 22.64 & 0.244 & 0.008 & 0.974 & 0.008 \\
\hline Palmitoleic acid (C16:1), \% & 2.58 & 2.21 & 2.04 & 2.13 & 1.96 & 1.84 & 0.058 & 0.100 & 0.001 & 0.001 \\
\hline Margaric acid (C17:0), \% & 0.28 & 0.33 & 0.27 & 0.30 & 0.27 & 0.24 & 0.013 & 0.028 & 0.064 & 0.004 \\
\hline Stearic acid (C18:0), \% & 11.64 & 10.04 & 10.45 & 11.81 & 10.10 & 10.49 & 0.196 & 0.940 & 0.579 & 0.001 \\
\hline Oleic acid $(\mathrm{C} 18: \ln 9 \mathrm{c}), \%$ & 44.45 & 40.69 & 40.74 & 42.36 & 39.96 & 40.20 & 0.307 & 0.035 & 0.001 & 0.001 \\
\hline Vaccenic acid (C18:1n7), \% & 0.55 & 0.51 & 0.42 & 0.65 & 0.47 & 0.37 & 0.070 & 0.469 & 0.891 & 0.020 \\
\hline Total C18:1 fatty acids, $5 \%$ & 44.87 & 41.05 & 40.93 & 42.76 & 40.25 & 40.37 & 0.301 & 0.033 & 0.001 & 0.001 \\
\hline Linoleic acid (C18:2n6c), \% & 12.96 & 19.65 & 19.71 & 16.08 & 20.01 & 20.38 & 0.246 & 0.001 & 0.001 & 0.001 \\
\hline Total C18:2 fatty acids, ${ }^{6} \%$ & 13.68 & 20.55 & 20.55 & 16.89 & 20.96 & 21.32 & 0.245 & 0.001 & 0.001 & 0.001 \\
\hline$\alpha$-Linolenic acid (C18:3n3), \% & 0.74 & 0.73 & 0.69 & 0.75 & 0.70 & 0.67 & 0.018 & 0.461 & 0.366 & 0.005 \\
\hline Eicosenoic acid (C20:1), \% & 0.51 & 0.81 & 0.81 & 0.68 & 0.86 & 0.85 & 0.018 & 0.002 & 0.001 & 0.001 \\
\hline Total SFA, ${ }^{7} \%$ & 37.09 & 34.10 & 34.51 & 36.37 & 34.82 & 34.56 & 0.282 & 0.052 & 0.946 & 0.001 \\
\hline Total MUFA, ${ }^{8} \%$ & 47.96 & 44.07 & 43.78 & 45.57 & 43.07 & 43.06 & 0.329 & 0.038 & 0.001 & 0.001 \\
\hline Total PUFA, ${ }^{9} \%$ & 14.55 & 21.46 & 21.40 & 17.73 & 21.72 & 22.12 & 0.244 & 0.001 & 0.001 & 0.001 \\
\hline UFA:SFA ${ }^{10}$ & 1.69 & 1.92 & 1.89 & 1.74 & 1.86 & 1.89 & 0.023 & 0.060 & 0.910 & 0.001 \\
\hline PUFA:SFA ${ }^{11}$ & 0.39 & 0.63 & 0.62 & 0.49 & 0.62 & 0.64 & 0.010 & 0.001 & 0.001 & 0.001 \\
\hline Iodine value, ${ }^{12} \mathrm{mg} / \mathrm{g}$ & 67.39 & 75.96 & 75.53 & 70.73 & 75.42 & 76.14 & 0.355 & 0.001 & 0.001 & 0.001 \\
\hline
\end{tabular}

${ }^{1}$ All items calculated as a percentage of the total fatty acid content. Fat samples were collected from the shoulder of each pig approximately $5 \mathrm{~cm}$ dorsal to the medial ridge of the scapula.

${ }^{2}$ Corn-soybean meal-based control diet with $0 \%$ dried distillers grains with solubles (DDGS), $0 \%$ wheat middlings (midds), and $0 \%$ corn oil.

${ }^{3}$ Control diet with $30 \%$ DDGS and $19 \%$ midds.

${ }^{4}$ Control diet with $3 \%$ corn oil.

${ }^{5}$ Total $\mathrm{C} 18: 1$ fatty acids $=[\% \mathrm{C} 18: \ln 9 \mathrm{c}]+[\% \mathrm{C} 18: 1 \mathrm{n} 7]$.

${ }^{6}$ Total $\mathrm{C} 18: 2$ fatty acids $=[\% \mathrm{C} 18: 2 \mathrm{n} 6 \mathrm{t}]+[\% \mathrm{C} 18: 2 \mathrm{n} 6 \mathrm{c}]+[\% \mathrm{C} 18: 2,9 \mathrm{c} 11 \mathrm{t}]+[\% 18: 2,10 \mathrm{t} 12 \mathrm{c}]+[\% \mathrm{C} 18: 2,9 \mathrm{c} 11 \mathrm{c}]+[\mathrm{C} 18: 2,9 \mathrm{t} 11 \mathrm{t}]$.

${ }^{7} \mathrm{Total} \mathrm{SFA}=[\% \mathrm{C} 14: 0]+[\% \mathrm{C} 15: 0]+[\% \mathrm{C} 16: 0]+[\% \mathrm{C} 17: 0]+[\% \mathrm{C} 18: 0]+[\% \mathrm{C} 20: 0]+[\% \mathrm{C} 21: 0]+[\% \mathrm{C} 22: 0]+[\% \mathrm{C} 24: 0]$.

${ }^{8}$ Total MUFA $=[\% \mathrm{C} 14: 1]+[\% \mathrm{C} 15: 1]+[\% \mathrm{C} 16: 1]+[\% \mathrm{C} 17: 1]+[\% \mathrm{C} 18: \ln 9 \mathrm{t}]+[\% \mathrm{C} 18: \ln 9 \mathrm{c}]+[\% \mathrm{C} 18: 1 \mathrm{n} 7]+[\% \mathrm{C} 20: 1]+[\% \mathrm{C} 24: 1]$.

${ }^{9}$ Total PUFA $=[\% \mathrm{C} 18: 2 \mathrm{n} 6 \mathrm{t}]+[\% \mathrm{C} 18: 2 \mathrm{n} 6 \mathrm{c}]+[\% \mathrm{C} 18: 29 \mathrm{c}, 11 \mathrm{t}]+[\% \mathrm{C} 18: 210 \mathrm{t}, 12 \mathrm{c}]+[\% \mathrm{C} 18: 29 \mathrm{c}, 11 \mathrm{c}]+[\% \mathrm{C} 18: 29 \mathrm{t}, 11 \mathrm{t}]+[\% \mathrm{C} 18: 3 \mathrm{n} 6]+[\%$ C18:3n3].

${ }^{10}$ UFA:SFA ratio $=[$ total MUFA + total PUFA $] /$ total SFA

${ }^{11}$ PUFA:SFA ratio $=$ total PUFA/total SFA.

${ }^{12}$ Iodine value $=[\% \mathrm{C} 16: 1] \times 0.95+[\% \mathrm{C} 18: 1] \times 0.86+[\% \mathrm{C} 18: 2] \times 1.732+[\% \mathrm{C} 18: 3] \times 2.616+[\% \mathrm{C} 20: 1] \times 0.785$.

diate. These changes in fatty acid profile, specifically decreases in total PUFA and carcass fat IV, suggest that withdrawing the DDGS and midds from the diet before harvest allowed for improved fat quality compared with feeding these ingredients to market; however, this approach did not return fatty acid concentrations to the same levels as those in pigs fed lowfiber, low-fat regimens throughout the entire study. Withdrawing these ingredients reduced the intake of PUFA provided in the diet; thus, the decrease in belly IV value is most likely related to PUFA intake rather than a direct effect of the fiber on PUFA profile. Similarly, Coble et al. (2014) found that jowl IV decreased linearly with increased withdrawal duration of DDGS and midds, but IV of pigs administered the longest withdrawal treatment of $20 \mathrm{~d}$ was still greater than that of pigs fed corn-soybean diets throughout the entire finishing period. Asmus et al. (2014) observed that switching pigs from diets containing DDGS and midds to a corn-soybean meal-based diet $23 \mathrm{~d}$ before market reduced jowl fat IV, but further reductions occurred when the withdrawal duration was extended to $47 \mathrm{~d}$ before harvest. In Exp. 2, when all diets types were fed for the entire duration of the trial, belly fat IV was increased by $8.2 \mathrm{~g} / \mathrm{g}$ for pigs fed diets with DDGS and wheat middlings and by $7.1 \mathrm{~g} / \mathrm{g}$ for pigs fed the corn oil diets compared with pigs fed the control.

Pelleting the diets increased UFA and carcass fat IV in both experiments, but the response to pelleting the different diet types was not consistent. In Exp. 1, the increase in PUFA and IV of belly fat in response to pelleting was greater when DDGS and midds were fed than when the corn-soybean meal diet was fed. The greater belly fat IV from pigs fed pelleted diets was 
unexpected, particularly because faster-growing pigs will have a lower IV than slower-growing pigs. Lo Fiego et al. (2005) reported that pigs with heaver BW and HCW had decreased PUFA and IV than lighter pigs. The reason for the increase in carcass fat IV is not entirely understood, but 1 hypothesis is that the pelleting process caused increased exogenous fat digestibility and, in turn, resulted in an increase in the amount of dietary oil that is deposited as carcass fat. Chae et al. (1997) reported that pelleting diets increased fat digestibility in finishing pigs. Thus, Exp. 2 was designed to test this hypothesis by including a diet with 3\% corn oil. Kim et al. (2013) reported that total tract true digestibility of acid-hydrolyzed ether extract is much greater for extracted corn oil than for the oil contained within DDGS (94.3\% vs. 51.9\%). Because fat from extracted corn oil is already highly digestible, we expected that pelleting would increase the digestibility of the fat from the DDGS and midds to a greater extent than the fat from corn oil. The results from Exp. 2, however, did not confirm this hypothesis. Pelleting the diets increased belly fat IV, regardless of diet type, and the interactions that occurred for fatty acid profiles of belly and shoulder fat were a result of pelleting increasing the PUFA levels to a greater extent for pigs fed the control diet than those fed the diet containing corn oil or the diet with DDGS and midds. Wondra et al. (1995) observed that feeding pelleted diets increased DM, N, and GE digestibility compared with feeding meal diets. The increase in carcass fat IV from pelleting may be related to the increased digestibility of nonfat nutrients in the diets, allowing fat to be deposited in the fatty acid form in which it is consumed. To our knowledge, the current trials are the first report of fatty acid change due to diet form.

In summary, pigs fed diets with DDGS and midds had poorer growth performance, decreased HCW, reduced carcass yield, and higher carcass fat IV than pigs fed the control diets. In Exp. 1, withdrawing DDGS and midds from the diet $17 \mathrm{~d}$ before market fully restored carcass yield to levels similar to the control-fed pigs, but only an intermediate improvement in belly fat IV was observed. The inclusion of ingredients with greater amounts of UFA increased carcass fat IV, regardless of source. Feeding pelleted diets increased ADG and improved G:F, but diet form did not influence HCW or carcass yield in either trial. In both experiments, feeding pelleted diets increased carcass fat IV; furthermore, the source of fat (endogenous from the ingredient vs. supplemental) in pelleted diets does not appear to affect the carcass fat IV response to pelleting.

\section{LITERATURE CITED}

American Oil Chemists' Society. 1998. Official methods and recommended practices of the AOCS. 5th ed. Am. Oil. Chem. Soc., Champaign, IL.

AOAC. 2006. Official methods of analysis. 18th ed. Assoc. Off. Anal. Chem., Arlington, VA.

ASAE. 1987. Wafers, pellets, and crumbles-Definitions and methods for determining density, durability, and moisture content. ASAE Standard S269.3. In: Agricultural engineers yearbook of standards. Am. Soc. Agric. Biol. Eng., St. Joseph, MI. p. 318.

ASAE. 1996. Cubes, pellets, and crumbles: Definitions and methods for determining density, durability, and moisture content. ASAE Standard S269.4. Am. Soc. Agric. Biol. Eng., St. Joseph, MI.

Asmus, M. D., J. M. DeRouchey, M. D. Tokach, S. S. Dritz, T. A. Houser, J. L. Nelssen, and R. D. Goodband. 2014. Effects of lowering dietary fiber before marketing on finishing pig growth performance, carcass characteristics, carcass fat quality, and intestinal weights. J. Anim. Sci. 92:119-128. doi:10.2527/ jas.2013-6679

Baird, D. M. 1973. Influence of pelleting swine diets on metabolizable energy, growth and carcass characteristics. J. Anim. Sci. 36:516-521.

Benz, J. M., S. K. Linneen, M. D. Tokach, S. S. Dritz, J. L. Nelssen, J. M. DeRouchey, R. D. Goodband, R. C. Sulabo, and K. J. Prusa. 2010. Effects of dried distillers grains with solubles on carcass fat quality of finishing pigs. J. Anim. Sci. 88:36663682. doi:10.2527/jas.2010-2937

Benz, J. M., M. D. Tokach, S. S. Dritz, J. L. Nelssen, J. M. DeRouchey, R. C. Sulabo, and R. D. Goodband. 2011. Effects of choice white grease and soybean oil on growth performance, carcass characteristics, and carcass fat quality of growing-finishing pigs. J. Anim. Sci. 89:404 413. doi:10.2527/jas.2009-2737

Chae, B. J., I. K. Han, J. H. Kim, C. J. Yang, S. J. Ohh, Y. C. Rhee, and Y. K. Chung. 1997. Effects of feed processing and feeding method on growth and carcass traits for growing-finishing pigs. Asian-Australas. J. Anim. Sci. 10:164-169.

Coble, K. F., J. M. DeRouchey, M. D. Tokach, R. D. Goodband, S. S. Dritz, T. A. Houser, B. Goehring, and M. J. Azain. 2014. Effects of withdrawing high-fiber ingredients before market on finishing pig growth performance, carcass characteristics, intestinal weights, and carcass fat quality. J. Anim. Sci. 92(Suppl. 2):86. (Abstr.)

Cromwell, G. L., M. J. Azain, O. Adeola, S. K. Baidoo, S. D. Carter, T. D. Crenshaw, S. W. Kim, D. C. Mahan, P. S. Miller, and M. C. Shannon. 2011. Corn distillers dried grains with solubles in diets for growing-finishing pigs: A cooperative study. J. Anim. Sci. 89:2801-2811. doi:10.2527/jas.2010-3704

De Jong, J. A., J. M. DeRouchey, M. D. Tokach, R. D. Goodband, S. S. Dritz, and J. L. Nelssen. 2013a. Effects of varying ingredient particle size and diet form on nursery pig growth performance and caloric efficiency. J. Anim. Sci. 91(Suppl. 2):70. (Abstr.)

De Jong, J. A., J. M. DeRouchey, M. D. Tokach, R. D. Goodband, S. S. Dritz, J. L. Nelssen, and C. Hastad. 2013b. Effects of corn particle size, complete diet grinding and diet form on pig growth performance, caloric efficiency, and carcass characteristics. J. Anim. Sci. 91(Suppl. 2):112. (Abstr.)

Gaines, A. M., J. D. Spencer, G. I. Petersen, and N. R. Augspurger. 2007. Effect of corn distiller's dried grains with solubles (DDGS) withdrawal program on growth performance and carcass yield in grow-finish pigs. J. Anim. Sci. 85(Suppl. 1):438. (Abstr.) 
Kim, B. G., D. Y. Kil, and H. H. Stein. 2013. In growing pigs, the true ileal and total tract digestibility of acid hydrolyzed ether extract in extracted corn oil is greater than in intact sources of corn oil or soybean oil. J. Anim. Sci. 91:755-763. doi:10.2527/ jas.2011-4777

Linneen, S. K., J. M. DeRouchey, S. S. Dritz, R. D. Goodband, M. D. Tokach, and J. L. Nelssen. 2008. Effects of dried distillers grains with solubles on growing and finishing pig performance in a commercial environment. J. Anim. Sci. 86:1579-1587. doi:10.2527/jas.2007-0486

Lo Fiego, D. P., P. Santero, P. Macchioni, and E. De Leonibus. 2005. Influence of genetic type, live weight at slaughter and carcass fatness on fatty acid composition of subcutaneous adipose tissue of raw ham in the heavy pig. Meat Sci. 69:107-114. doi:10.1016/j.meatsci.2004.06.010

McClelland, K. M., G. Rentfrow, G. L. Cromwell, M. D. Lindemann, and M. J. Azain. 2012. Effects of corn distillers dried grains with solubles on quality traits of pork. J. Anim. Sci. 90:4148-4156. doi:10.2527/jas.2011-4779

Myers, A. J., R. D. Goodband, M. D. Tokach, S. S. Dritz, J. M. DeRouchey, and J. L. Nelssen. 2013. The effects of diet form and feeder design on the growth performance of finishing pigs. J. Anim. Sci. 91:3420-3428. doi:10.2527/jas.2012-5612

Nemechek, J., M. Tokach, E. Fruge, E. Hansen, S. Dritz, R. Goodband, J. DeRouchey, and J. Nelssen. 2012. Effects of pellet quality and feeder adjustment on growth performance of finishing pigs. J. Anim. Sci. 91(Suppl. 2):215. (Abstr.)

NRC. 2012. Nutrient requirements of swine. 11th rev. ed. Natl. Acad. Press, Washington, DC.
Salyer, J. A., J. M. DeRouchey, M. D. Tokach, S. S. Dritz, R. D. Goodband, J. L. Nelssen, and D. B. Petry. 2012. Effects of dietary wheat middlings, distillers dried grains with solubles, and choice white grease on growth performance, carcass characteristics, and carcass fat quality of finishing pigs. J. Anim. Sci. 90:2620-2630. doi:10.2527/jas.2011-4472

Stark, C. R., K. C. Behnke, J. D. Hancock, and R. H. Hines. 1993. Pellet quality affects growth performance of nursery and finishing pigs. In: Proc. Swine Day. Stn. Rep. Prog. No. 695. Agric. Exp. Stn. Coop. Ext. Serv., Kansas State Univ., Manhattan. p. 71-74.

Stein, H. H., and G. C. Shurson. 2009. Board-invited review: The use and application of distillers dried grains with solubles in swine diets. J. Anim. Sci. 87:1292-1303. doi:10.2527/ jas.2008-1290

Widmer, M. R., L. M. McGinnis, D. M. Wulf, and H. H. Stein. 2007. Effects of co-products from the ethanol industry on pig performance and carcass composition. J. Anim. Sci. 85(Suppl. 1):437. (Abstr.)

Wondra, K. J., J. D. Hancock, K. C. Behnke, R. H. Hines, and C. R. Stark. 1995. Effects of particle size and pelleting on growth performance, nutrient digestibility, and stomach morphology in finishing pigs. J. Anim. Sci. 73:757-763.

Xu, G., S. K. Baidoo, L. J. Johnston, D. Bibus, J. E. Cannon, and G. C. Shurson. 2010. The effects of feeding diets containing corn distillers dried grains with solubles, and withdrawal period of distillers dried grains with solubles, on growth performance and pork quality in grower-finisher pigs. J. Anim. Sci. 88:1388-1397. doi:10.2527/jas.2008-1403 
Reproduced with permission of the copyright owner. Further reproduction prohibited without permission. 